\title{
GenYacht: An Interactive Generative Design System for Computer-Aided Yacht Hull Design
}

\author{
Shahroz Khan ${ }^{* 1}$ Erkan Gunpinar ${ }^{2}$ Bekir Sener ${ }^{3}$ \\ ${ }^{1}$ University of Strathclyde, Glasgow, United Kingdom \\ 2 Istanbul Technical University, Istanbul, Turkey \\ 3 Yildiz Technical University, Istanbul, Turkey
}

\begin{abstract}
In the present work, a new digital design system, GenYacht, is proposed for the creation of optimal and user-centred yacht hull forms. GenYacht is a hybrid system involving generative and interactive design approaches, which enables users to create a variety of design alternatives. Among them, a user can select a hull design with desirable characteristics based on its appearance and hydrostatics/hydrodynamic performance. GenYacht first explores a given design space using a generative design technique (GDT), which creates uniformly distributed designs satisfying the given design constraints. These designs are then presented to a user and single or multiple designs are selected based on the user's requirements. Afterwards, based on the selections, the design space is refined using a novel space-shrinking technique (SST). In each interaction, SST shrinks the design space, which is then fed into GDT to create new designs in the shrank space for the next interaction. This shrinkage of design space guides the exploration process and focuses the computational efforts on user-preferred regions. The interactive and generative design steps are repeated until the user reaches a satisfactory design(s). The efficiency of GenYacht is demonstrated via experimental and user studies and its performance is compared with interactive genetic algorithms.
\end{abstract}

Keywords: Generative Design, Interactive Design, Computer-Aided Design, Yacht Hull Design

\section{Introduction}

The arrival of the fourth industrial revolution, Industry 4.0, has transformed the traditional design and manufacturing techniques. There has been an uprise in the efforts by various industries in digitalisation and smartness of design systems, which harvest the computational power to explore design spaces for ingenious, optimal and user-centred designs/solutions. However, even with such advancements, the maritime industry is still based on relatively traditional and passive computational design techniques. In general, naval architects retrieve single or multiple parent hull forms and apply mirror adjustments to obtain a new form with desired characteristics, whose design performance is usually checked in the end by simulation. If the results are not satisfactory, these steps are repeated iteratively until the design and performance requirements are fulfilled. This is a trial-and-error method and highly dependent on the experience of the designer. Such an exploration of design space cannot guarantee the generation of true optimal design and fails to approximate the design space well. Furthermore, the nonintuitive nature of these techniques cannot capture the naval architects' design intention. Although, some academic scholars from the maritime field have made a considerable amount of contribution to the modernisation of preliminary ship design

*Email: shahrozkhan2020@gmail.com, shahroz.khan@starth.ac.uk Address: University of Strathclyde, Glasgow, United Kingdom, Tel: +44 (0)7405 815527 techniques, however, their usage in the industry is still limited. Some of the recent efforts to support ship design at the preliminary stage includes the development of attribute-based design techniques [1]; parametric design systems [2]; library-based [3], sketching based [4], interactive optimisation [5] based and three-dimensional packing based $[6,7]$ approaches for exploration of hull form variations; simulation-driven [8] and holistic approach to ship design [9] and machine learning-based ship design method to assist the optimisation towards the optimal solution [10].

In this paper, we aim to take the next step in the computeraided preliminary yacht hull design by interactively inducing the user preference on designs into the design space exploration. This is achieved by introducing a new interactive design system, GenYacht, which brings the benefit of the interactive and generative design to the preliminary design stage to generate user-driven hull forms with better performance. However, the proposed interactive technique can also be utilized for different design applications in maritime and other engineering fields.

Generative design is an algorithm-driven design process to empower experienced or novice designers to generate the desired number of optimum alternatives for an initial design. Instead of a single solution, the generative design creates potentially various solutions satisfying the given design requirements and facilitates the designer with the comfort of selecting a solution that best satisfies his/her needs [11]. As even for the most experienced designers, their intuition might be limited when 
manually exploring an unprecedented large design space. In generative design, a basic layout of an input CAD model is first created. Design specifications and constraints are then defined. Various computational simulations are later executed to obtain a set of optimised solutions [12].

Interactive design is a process in which a given design space is explored, and a target design is evaluated based on human subjective evaluation. The interaction with a human evaluator facilitates the generation of a solution that incorporates human intuition without explicitly codifying them into the design process. In interactive design systems, user carry out the design exploration either with interactive interfaces $[13,14,15,16]$ or by integrating the meta-heuristics with the interactive interfaces to semi-automate the exploration process $[5,17,18,19]$. In the latter approaches, users are interactively involved at each iteration/generation of an optimiser and guide the optimisation process towards the promising regions of the design space. In this approach, an initial population is first created consisting of randomly sampled designs, and a user then performs interaction for selecting a design [20] or he/she can rate all the designs shown [21]. The optimiser then performs an iteration to generate designs similar to the selected or highly-rated de$\operatorname{sign}(\mathrm{s})$. The creation of similar designs is usually done utilising a distance-based metric $[17,19]$. This iterative and interactive process continues until the user reaches a preferred or satisfactory design.

As designs generated in each iteration are based on the user's selection(s) in the previous iteration, starting the interactive process with the randomly generated designs, which are mostly clustered and non-uniformly distributed, can restrain the user from exploring all the design possibilities. Furthermore, distance-based exploration can force the optimiser to converge to similar designs at a fast rate; therefore, a large portion of design space can be left unexplored, which will be proven via experimentations later in this work.

The proposed system is based on novel interactive and generative design techniques, which run in parallel during the hull form creation. The generative design technique (GDT) provides a promising way to explore the design space, and to generate well-diverse design alternatives automatically. A design space is first created based on the upper and lower bounds of geometric parameters of the parent hull. In this space, GDT then generates a set of $N$ design alternatives. These alternatives are uniformly distributed in the design space, and each design represents a particular location in the design space (see the output of GDT in Figure 1, which illustrates the hull forms generated in two-dimensional design space). The interactive design involves user preference/intuition interactively during the design process, thereby guiding the design exploration towards a more promising region of design space. At this step, hull designs are searched with GDT, and three-dimensional (3D) surface models for the yacht hulls are generated using Khan et al.'s [22] parametric design technique. Afterwards, these models are presented to the user along with their physical properties such as hydrostatics and resistance. The user then performs interaction while selecting a design(s) and design space is then refined based on the chosen design(s). An overall workflow of GenY- acht is shown in Figure 1. In this work, the refinement of the design space is done using a novel space-shrinking technique (SST), which shrinks the design space and generates new designs in the shrunk space for the next interaction. The interactive process continues until the user reaches a hull design with desired characteristics. It is noteworthy that the user selections are made not only based on the performance of the hull but also according to its form appearance.

\section{Related works}

Triggered by advances in digital design and manufacturing, interactive and generative design has received significant attention in computer-aided design (CAD) and computer graphics communities. We mainly focus our literature review on the interactive and generative design for the exploration of design space for parametric CAD shapes. In this section, we first review prior works in interactive design, followed by a discussion of existing studies in naval architecture and a brief introduction of generative design systems.

\subsection{Interactive Design}

Computational design tools help users to create digital designs for various applications, which is done using optimisation techniques, interactive interfaces or a combination of both to create hybrid systems. These tools guide users into exploring a given parametric design space for certain physical criteria. Mostly, interactive interfaces (commonly used in computer graphics community) are developed for specific design applications, which are used to synthesise and assemble components to explore design variations. For instance, Bole [13] developed a transformation tool to interactively manipulate geometric parameters for a ship hull design. Interactive tools have also been proposed for synthesising three-dimensional (3D) characters [23], procedural modelling of the architectural structures [24] and for 3D modelling of garment patterns [25]. Some researchers have also developed interactive techniques for exploring 3D shape variations [26, 27] and for prediction of their physical properties, such as the aerodynamics of automotive [16] and mechanical stress of 3D components [14].

Interactive design approaches have also been coupled with meta-heuristics, which usually refer as Interactive Evolutionary Computation (IEC). In IEC, human evaluation is used as a component of objective function during the solution space exploration for an optimum solution. During exploration, the user's intuitive assessment of a solution is incorporated to create a user-oriented or user-centred design. During optimisation, the incorporation is carried out in different ways and for different end objectives. In IEC, an overwhelming majority of works proposed interactive genetic algorithms (IGA) for various design applications. IGAs are based on the principle of the typical genetic algorithm (GA). Brintrup et al. [17] proposed an IGA to incorporate the qualitative and quantitative criteria for ergonomic chair design. In their technique, a user plays the role of the qualitative criterion guiding the optimisation to the desired location in the design space. First, an initial population of solutions are presented to the user, where he/she rates 


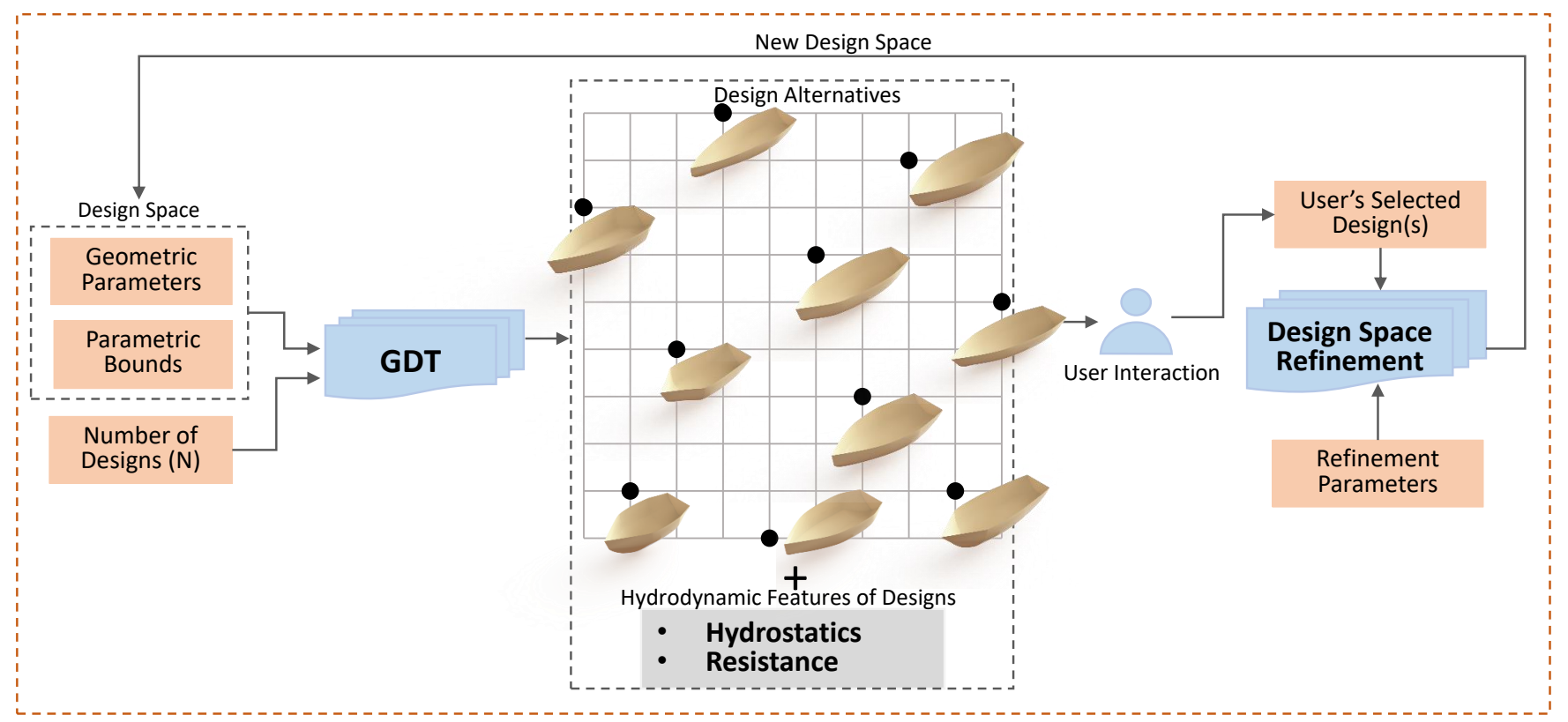

Repeat Until Desired Design is Achieved

Figure 1: Overall workflow of GenYacht. A design space, formed with geometric parameters and their limits, is inputted into the generative design technique (GDT), which generates $N$ uniformly distributed hull design alternatives. Among them, a user selects a design based on its appearance and physical properties such as hydrostatics and resistance. The design space is then refined according to the selected designs, which is again fed into GDT for creating new designs. This process is repeated until the final design(s) is achieved.

the designs on a Likert scale, and these ratings act as a fitness value for each design. Therefore, the designs with higher scores become parents, and the evolutionary process is carried out to generate designs similar to the parent. A multi-stage IGA (MSIGA) was proposed by Dou et al. [21]. At the initial stage, MS-IGA generates populations of simple designs and as the interactive evolutionary process continues the design becomes complex. Dou et al. argue that this helps to minimise the user fatigue during an interaction, which is one of the major drawbacks of the IGA based systems [28]. In IEC, user fatigue is the inability of the user to select potential designs during the design interaction due to physical or psychological exhaustion [17]. In another study, along with the user rating, Dou et al. [29] incorporated the time spent to evaluate each design to calculate its fitness value. The incorporation of evaluation time simulates the user hesitancy into the design process. The performance of their works $[21,29]$ was validated with a car dashboard design.

Poirson et al. [19] elicited user perception about the product design using IGA. In their approach, a user first selects the design which mostly represents a given semantic attribute. Then, GA moves the population of solutions closer to the selected design via a distance function. Poirson et al. also performed different experiments on the parameter tuning of GA, as the convergence of GA mainly depends on these tuning parameters. Hernandez et at. [30] addressed the problem of Unequal Area Facility Layout using an IGA. However, instead of presenting the entire population of designs, Hernandez et al. [30], and Machwe and Parmee [31] utilised clustering techniques to enable a user to evaluate the representative design of each cluster, thereby ameliorating user fatigue. Gu et al. [32] incorporated Neural Network-based learning technique, General Regression
Neural Network (GRNN), into IEC to approximate the user's aesthetic perception during the interactive evolutionary process. IGA-based systems have also been proposed for aircraft [33], software design [34] and structural design [35].

The literature also contains some recent techniques [36] to interactively prune the Pareto Front solution set at each generation of multi-objective GA, which helps to reduce the size of the Pareto front and to obtain the desired Pareto optimal solutions at the end of the evolutionary process. Recently, few researchers have diverted also their attention in utilising other meta-heuristic algorithms such as Particle Swarm [20] and Teaching-Learning-Based Optimisation [18] to develop IECbased design systems.

IGA has been used for various design applications, however, to the best of our knowledge, Duchateau's work [5] is the only example related to the subject of the present study in the field of naval architecture. In [5], Duchateau proposed an IGA-based technique to allow users to create and select designs based on insight gained during the design exploration process. The proposed technique was applied for the preliminary design of a mine-countermeasures vessel. Duchateau argued that the complexity of the ship design hinders designer to explore vast and potentially more region of the design space with traditional design techniques. Therefore, an interactive and evolutionary approach was proposed to gradually steer the optimiser to the exploration of more promising design solutions. In naval architecture, DeNucci's work [37] is another example of the involvement of user into the design process, which focuses on capturing and integration of the design rationale (i.e., reasoning behind the design decision) at the conceptual stage of ship design. DeNucci developed a Rationale Capture Tool (RCT) 
to incorporate design rationale as user experience and performance into the design process, thereby linking the users' design configuration preferences to the ship performance at its preliminary design phase.

In this work, we aim to propose a new interactive design to overcome the aforementioned drawbacks of IGA-based system. Therefore, in Table 1, we describe some advantages of GenYacht over typical IGA-based design systems.

\subsection{Generative Design}

During the last few years, generative design techniques have played a critical role in automating the exploration of parametric design spaces. Unlike traditional optimisation based design exploration, GDTs explore large design spaces to find a variety of optimal alternatives that give users the ability to choose a design that best fits his/her needs. Literature contains many efforts from researchers in design exploration techniques for preliminary design of naval vessels $[7,38,9,10]$. However, these techniques are not developed in the context of generative design and therefore, can only explore a limited region of design space to generate single or Pareto designs, which are usually a slight variation of the parent shape. To give some background to the readers from naval architecture, here, we mentioned some existing generative design systems developed for parametric design exploration and their limitations.

A random search based generative system, Genoform, was developed by Krish et al. [11] for parametric design exploration, in which variation between designs is achieved via Euclidean distance-based similarity criterion. Genoform cannot explore a design space well due to its random search nature, which has been proven via experimentation in [12]. An iterative design exploration system, Fractal [39], was developed by Autodesk, which provides $n^{I}$ design possibility for a given parametric shape. Here, $n$ represents the geometric parameters and $I$ is the number of levels for each parametric range. Another system called Dream Lens was proposed by Matejka et al. [40] to explore and visualise a large number of generatively created designs. Dream Lens explores performance spaces for the given problem domains. Recently, Khan et al. [41] proposed a Psycho-physical distance metric to induce human perception into the design process for the exploration of diverse shapes.

Similarly, Kazi et al. [42] developed DreamSketch, a generative design platform for the exploration of design sketches at the conceptual stage. The usability of this system requires users to have digital sketching skills. Moreover, Zaman et al. [43] devised GEM-NI, which is a generative design software for design exploration of two-dimensional shapes. Later, an extension of GEM-NI called MACE was also proposed by Zaman et al. [44] with enhancing capability of visualisation of design alternatives. Gunpinar and Gunpinar [45] proposed a generative design approach based on a particle tracing algorithm, and recently, Khan and Awan [12] developed a generative design system, DesignN, for exploration of CAD shapes with continuous and discrete parameters. However, in [45] and [12] no physical performance criterion was evaluated during the design exploration.

Some researchers have also introduced some applicationspecific generative design systems, such as ParaGen, Dexen and GENE_ARCH, which were introduced by Turrin et al. [46], Patrick [47] and Caldas [48] for exploring parametric structures, façade and energy efficient building designs, respectively.

\section{Method Overview}

In this section, the algorithmic details of Gen Yacht will be introduced. After describing the basic terminology and the generative design approach, the proposed interactive design approach in line with the space-shrinking technique and Gen Yacht's userinterface will be introduced.

\subsection{Basic Terminology and Generative Design Techniques (GDT)}

Let a design space $\mathcal{X}$ formed for a parent yacht hull $m$, which is represented using a set of geometric parameters, $\mathbf{x}_{m}=$ $\left\{x_{m, k}, k=1,2, \ldots, n\right\} \in \mathcal{X} \subseteq \mathbb{R}^{n} . \mathcal{X}$ is a subset of $\mathbb{R}^{n}$ and is bounded by the lower $\mathbf{x}_{\mathbf{m}}^{\mathbf{l}}$ and upper $\mathbf{x}_{\mathbf{m}}^{\mathbf{u}}$ bounds of geometric parameters (i.e. $\mathcal{X}:=\left\{x_{m, k}^{l} \leq x_{m, k} \leq x_{m, k}^{u}, \forall k \in\{1,2, \ldots n\}\right\}$ ).

It is impractical, if not impossible, for a user to manually iterate through all the astronomical possibilities of hull designs in $\mathcal{X}$. Therefore, our objective is to explore $\mathcal{X}$ with the aid of an optimiser to find a set $\mathcal{N}$ consisting of $N$ uniformly distributed diverse hull forms $\left(\mathcal{N}=\left\{\mathbf{x}_{1}, \mathbf{x}_{2}, \mathbf{x}_{3}, \ldots, \mathbf{x}_{N}\right\} \in \mathcal{X}\right)$. Here, $N$ is a user-defined parameter and each design in $\mathcal{N}$ represents a specific location in $\mathcal{X}$. To obtain the set $\mathcal{N}$, Khan and Gunpinar's approach [49] is adopted, which is briefly explained in this subsection. This approach utilises Audze and Eglais [50] space-filling criterion $\left(F_{1}(\mathcal{N})\right)$ to find uniformly distributed designs (see Equation 1).

$$
F_{1}(\mathcal{N})=\sum_{p=1}^{N-1} \sum_{q=p+1}^{N} \frac{1}{\mathcal{D}\left(\mathbf{x}_{p}, \mathbf{x}_{q}\right)^{2}}
$$

where

$$
\mathcal{D}\left(\mathbf{x}_{p}, \mathbf{x}_{q}\right)=\sqrt{\sum_{k=1}^{n}\left(x_{p, k}-x_{q, k}\right)^{2}}
$$

Here, $\mathcal{D}\left(\mathbf{x}_{p}, \mathbf{x}_{q}\right)$ is the Euclidean distance between the designs $p$ and $q$. Minimisation of $F_{1}(\mathcal{N})$ favours the uniform distribution of the $N$ designs in $\mathcal{X}$.

In the case of high-dimensional design spaces, the spacefilling criterion favours the placement of designs to the design space's boundaries, which is undesirable. Therefore, the spacefilling designs are searched within the class of Latin-hypercube with a criterion of non-collapsingness between designs. This criterion divides each dimension of $\mathcal{X}$ into $N$ intervals and ensures that two designs do not share the same range. It is incorporated into the search process using Equation 3, which calculates the number of intervals that $N$ designs share. Minimising this equation creates either complete or semi non-collapsing designs depending on a user-controlled parameter $\Omega$, which adjusts the weight for $F_{2}(\mathcal{N})$.

$$
F_{2}(\mathcal{N})=\Omega \times \sum_{p=1}^{N-1} \sum_{q=p+1}^{N} \mathcal{K}\left(\mathbf{y}_{p}, \mathbf{y}_{q}\right)
$$


Table 1: Comparison between IGA-based systems and GenYacht

\begin{tabular}{|c|c|c|}
\hline No. & IGA & GenYacht \\
\hline 1 & $\begin{array}{l}\text { Interactive process starts with the initial population of ran- } \\
\text { domly generated designs, which, in most cases, are not } \\
\text { well spread out in the design space. Therefore, this can } \\
\text { limit users from well exploring all regions of design space } \\
\text { [27]. }\end{array}$ & $\begin{array}{l}\text { The interactive process starts with uniformly distributed } \\
\text { designs, covering all the design possibilities within the } \\
\text { design space. This allows users to effectively explore the } \\
\text { entire design space. }\end{array}$ \\
\hline 2 & $\begin{array}{l}\text { Requires tuning of optimisation parameters, such as se- } \\
\text { lection operator, crossover and mutation probability, for } \\
\text { desirable results, which is non-trivial for most of the users } \\
\text { [12]. }\end{array}$ & $\begin{array}{l}\text { Does not require parameter tuning of the optimization spe- } \\
\text { cific parameters. The only user-defined parameter is the } \\
\text { shrink/expand rate, which controls the diversity of designs } \\
\text { in each interaction. According to the experiments con- } \\
\text { ducted in this work, shrink/expand rate does not affect the } \\
\text { performance of the optimizer to generate uniformly dis- } \\
\text { tributed and non-collapsing designs. }\end{array}$ \\
\hline 3 & $\begin{array}{l}\text { Selection of suitable distance metric(s) is critical [19] to } \\
\text { converge (i.e., get similar) the initial population of solu- } \\
\text { tions towards the user-selected designs. }\end{array}$ & $\begin{array}{l}\text { The designs space is shrunk in each interaction while } \\
\text { eliminating the non-preferred regions, which aids the op- } \\
\text { timiser to converge to the user-preferred designs. }\end{array}$ \\
\hline 4 & $\begin{array}{l}\text { It is hard to maintain the high variations between designs } \\
\text { in the interactions. }\end{array}$ & $\begin{array}{l}\text { A user can create significantly diverse designs at each } \\
\text { interaction using space-filling and non-collapsing criteria } \\
\text { (will be discussed in Section 3). }\end{array}$ \\
\hline 5 & $\begin{array}{l}\text { Starting the interactive process with random designs re- } \\
\text { quires users to carry out a large number of interactions to } \\
\text { explore all the design possibilities. Therefore, this higher } \\
\text { number of design evaluations can increase user fatigue } \\
{[17,31] \text {, thereby resulting in converging towards the lo- }} \\
\text { cal optimal and undesirable solutions. }\end{array}$ & $\begin{array}{l}\text { Starting the interactive process with uniformly distributed } \\
\text { design can help users to explore more design possibilities } \\
\text { with fewer design evaluations, which reduces the possi- } \\
\text { bility of user fatigue. Moreover, the space-shrinking tech- } \\
\text { nique provides better control of the total number of inter- } \\
\text { actions to be performed. }\end{array}$ \\
\hline
\end{tabular}

$$
\begin{gathered}
\mathcal{K}\left(\mathbf{y}_{p}, \mathbf{y}_{q}\right)=\sum_{j=1}^{n} f\left(y_{p, k}, y_{q, k}\right) \\
f\left(y_{p, k}, y_{q, k}\right)= \begin{cases}1 & \text { if } y_{p, k}=y_{q, k} \\
0 & \text { otherwise }\end{cases}
\end{gathered}
$$

In Equation 3, $\mathcal{K}\left(\mathbf{y}_{p}, \mathbf{y}_{q}\right)$ denotes the number of intervals that the designs $p$ and $q$ share, and $\mathbf{y}_{p}$ and $\mathbf{y}_{q}$ are the discrete representations for $\mathbf{x}_{p}$ and $\mathbf{x}_{q}$, respectively. To calculate the discrete value $\left(y_{p, k}\right)$ of $k^{\text {th }}$ geometric parameter $\left(x_{i, k}\right)$ for the $i^{\text {th }}$ design, its range between lower $\left(x_{i, l}^{k}\right)$ and upper $\left(x_{i, u}^{k}\right)$ bounds is first partitioned into $N$ intervals $\left[x_{i, k}^{l}=x_{i, k}^{1}, x_{i, k}^{2}, \ldots, x_{i, k}^{N}=x_{p, k}^{u}\right]$ and an integer coordinate $r$ is then assigned to $y_{i}^{k}$ as follows: $\forall r \in\{1,2, \ldots, N\},\left(x_{i, k}^{r} \leq x_{i, k}<x_{i, k}^{r+1}\right) \Rightarrow\left(y_{i, k}=r\right)$.

During the design exploration for the $N$ designs, the cost function $\mathbf{F}(\mathcal{N})$ in Equation 6 is minimised.

$$
\mathbf{F}(\mathcal{N})=\sum_{p=1}^{N-1} \sum_{q=p+1}^{N} \frac{1}{\mathcal{D}\left(\mathbf{x}_{p}, \mathbf{x}_{q}\right)^{2}}+\Omega \times \sum_{p=1}^{N-1} \sum_{q=p+1}^{N} \mathcal{K}\left(\mathbf{y}_{p}, \mathbf{y}_{q}\right)
$$

In this approach, design exploration process starts by generating an initial population (P) consisting of $N$ sub-populations $\left(\mathbf{P}=\left\{p_{L}, L=1,2, \ldots N\right\}\right)$. The $L^{\text {th }}$ sub-population of $\mathbf{P}$ consists of $s$ randomly sampled designs $\left(p_{L}=\left\{\mathbf{x}_{g}, g=1,2, \ldots s\right\}\right)$ in $\mathcal{X}$. This means for each solution, $\mathbf{P}$ contains a sub-population of size $s$. During the convergence, an optimiser guides all the subpopulations to their optimum position under the consideration of each sub-population's best solution (i.e. a solution that minimise $\mathbf{F}(\mathcal{N})$ ). Initial solution set $\mathcal{N}=\left\{\mathbf{x}_{p_{1}}, \mathbf{x}_{p_{2}}, \ldots, \mathbf{x}_{p_{N}}\right\}$ is first obtained from $\mathbf{P}$ containing $N$ solution; one solution from each sub-population using a greedy-selection strategy [49]. The initial $\mathcal{N}$ contains the combination of solutions which gives minimum value of $\mathbf{F}(\mathcal{N})$. During the optimisation, each iteration is completed by performing $N$ sub-iterations, and a sub-iteration is completed after updating all the designs in a sub-population using an optimiser. After the convergence, GDT returns an optimal set $\mathcal{N}_{o p}$ of $N$ space-filling designs. Algorithm 1 summarises the stepwise procedure of GDT.

Different optimisers, such as Genetic Algorithm (GA) [51], Particle Swarm Optimisation (PSO) [52], Artificial Bee Colony (ABC) [53], Teaching-Learning-Based Optimisation (TLBO) [54] and Jaya Algorithm (JA) [55], have been integrated into GDT and a final selection for Gen Yacht system was made based on optimisers' performance and computational complexities. The results of these optimisers will be shown in Section 4.1. Figure 2 (a) and (b) show the randomly distributed designs and uniformly distributed designs created using GDT. It can be seen that designs generated using GDT are well distributed and cover all the regions of the design space.

\subsubsection{Constrained Design Spaces}

Gen Yacht also gives users the ability to interactively explore constrained spaces, which are composed of feasible (i.e. designs satisfying the constraints) and infeasible (i.e. designs violating the constraints) designs. GDT should only generate feasible designs. Therefore, in this work, Deb's heuristic 


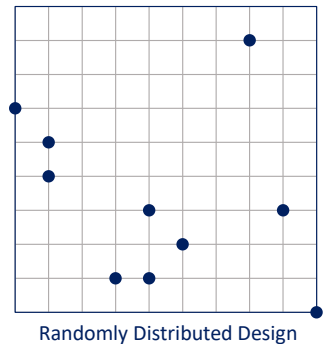

(a)

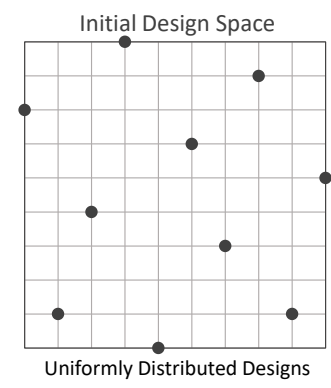

(b)

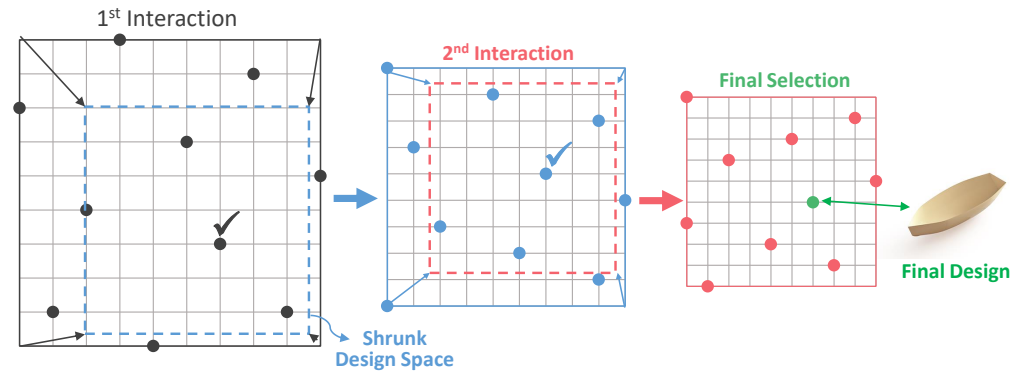

(c)

Figure 2: Illustration of designs generated via random sampling in a two-dimensional design space (a). While the designs generated using GDT in the same two-dimensional space are uniformly distributed because of space-filling and non-collapsing criteria (b). The interaction process started with GDT generated designs and in each interaction, design space is shrunk towards the user selection (c). The selection of design is indicated with a tick-mark.

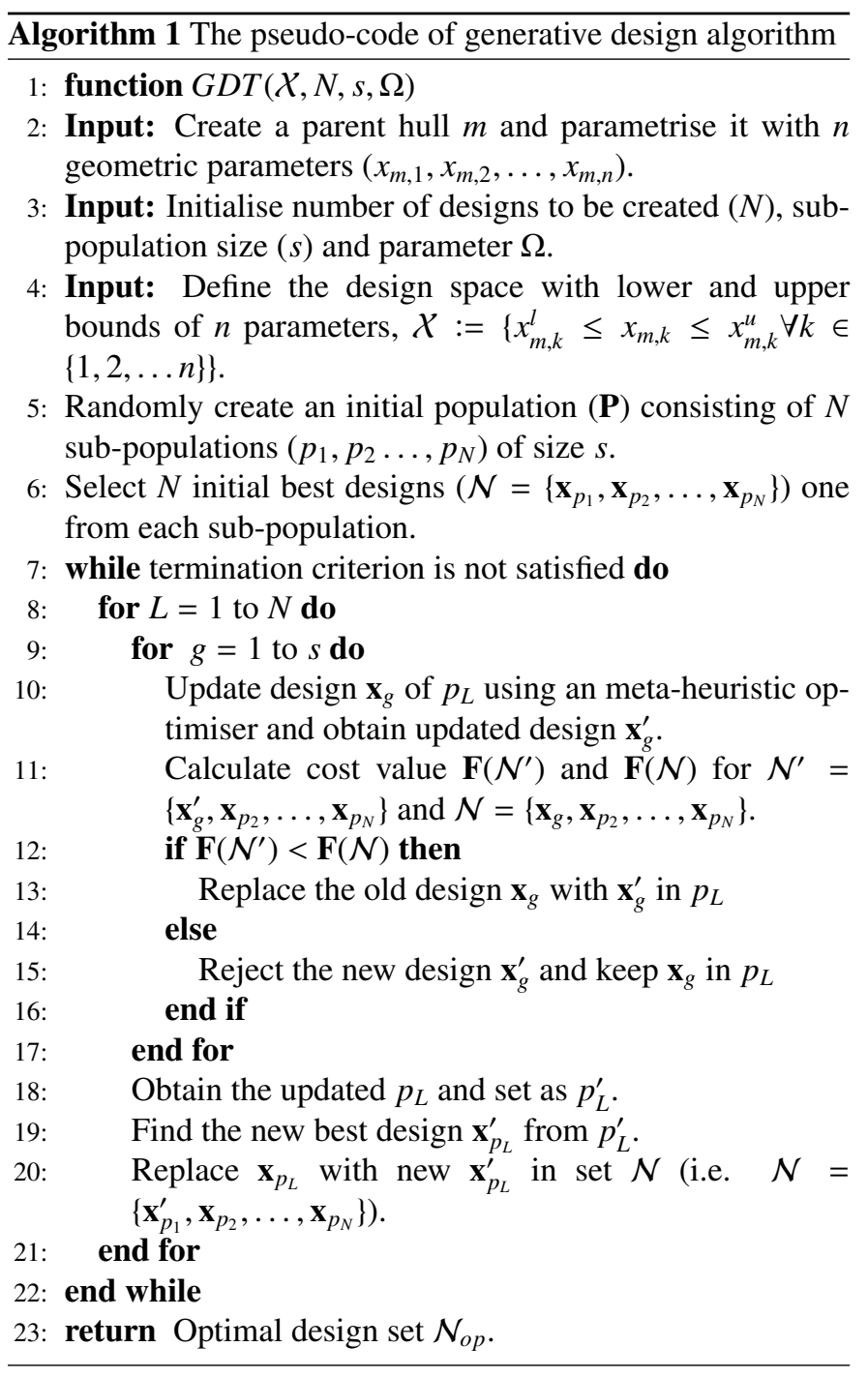

constrained handling method [56] was utilised, which uses a tournament selection operator. This operator selects two designs and compares them with each other. A design $p$ is said to be constrained-dominate other design $q$ if any of the following heuristic rules are true:

1. The design $p$ is feasible and design $q$ is not.

2. The designs $p$ and $q$ both are infeasible, but design $p$ vio- late less number of constraints.

3. The designs $p$ and $q$ both are feasible, but design $p$ has minimum cost function value.

The design $p$ is selected only if it constrained-dominate design $q$. In case, if both designs, $p$ and $q$, are infeasible and have the same number of constraint violations, the design with better cost value is then selected.

\subsection{Interactive Design Approach}

In an interactive design stage, $N$ hull designs generated via GDT are shown to the user along with their physical properties such as form coefficients, residuary and frictional resistance, metacentric radius, metacentre, longitudinal and transverse moments of inertia, longitudinal and vertical centre of buoyancy and flotation. The user then selects the designs according to the hulls' overall appearance and physical properties. This interaction step allows users to compare designs based on their design requirements and helps to make an appropriate design decision. Once the desired hull form is selected, the design space is refined based on the selected design. The refined design space is then imported into GDT to generate new designs in the next interaction step. This interaction procedure is repeated multiple times until a desirable number of designs are obtained. Figure 2 (c) illustrates the implementation of proposed interactive design approach on a two-dimensional design space. As shown in this Figure, at each interaction, the design space formed in the previous interaction shrinks by focusing on the preferred designs. In this way, the region of the user's interest can be better scanned.

Our design space shrinking process follows the analogy of woodcarving in which a carver first selects a large piece of timber (usually bigger than the size of the final form) to create the desired artefact. He/she then removes the large chunks of wood to achieve a general shape. Afterwards, the carver scrapes the pieces of wood step-by-step and gradually proceeds to a final shape. Such material scraping can be reflected as an exponential decay. In the initial interactions, the design space shrinks at a faster rate, and it decreases exponentially as the interaction process continues. The algorithmic details of the proposed SST are given in the next section. 


\subsubsection{Space-Shrinking Technique (SST)}

In the proposed interactive approach, a initial design set $\mathcal{N}_{o p}$ is first generated using GDT and an interaction loop between the user and GenYacht is then completed involving three steps: (2) the user selects preferable design(s) among the ones generated by GDT, (3) the design space is refined based on the selection(s), and (3) the shrunk space is inputted to GDT for the creation of new designs )for the next interaction. At the end of the multiple interaction loops, single or multiple preferred designs are obtained.

For the sake of simplicity, a single hull selection will be considered in the method's explanations. In the $T^{\text {th }}$ interaction loop, the user selects a $t^{\text {th }}$ design $\left(\mathbf{x}_{t}\right)$ from $\mathcal{N}_{o p}^{T-1}=$ $\left[\mathbf{x}_{p_{1}}, \mathbf{x}_{p_{2}}, \ldots, \mathbf{x}_{p_{N}}\right]$, which is obtained from GDT in the $(T-1)^{\text {th }}$ ( $T$ is integer) interaction. After the user selection, a new design space, $X^{T}$, is formed while shrinking the previous design space $\left(\mathcal{X}^{T-1}\right)$ based on the selected/preferred design $\mathbf{x}_{t}=$ $\left(x_{t, 1}, x_{t, 2}, \ldots, x_{t, n}\right)$, and a new design set $\mathcal{N}_{o p}^{T}$ is obtained from $X^{T}$. The shrinking of design space is performed by calculating new lower $\left(\hat{\mathbf{x}}_{\mathbf{m}}^{\mathbf{l}}\right)$ and upper $\left(\hat{\mathbf{x}}_{\mathbf{m}}^{\mathbf{u}}\right)$ bounds using Equation 7 for $\mathcal{X}^{T}:=\left\{\hat{x}_{m, k}^{l} \leq x_{t, k} \leq \hat{x}_{m, k}^{u}, \forall k \in\{1,2, \ldots n\}\right\}$.

$$
\left\{\begin{array}{l}
\dot{x}_{m, k}^{l}=x_{m, k}^{l}+\left|\lambda_{-}^{T} \times \mathcal{R}_{T}^{l}\right| \\
\dot{x}_{m, k}^{u}=x_{m, k}^{u}-\left|\lambda_{-}^{T} \times \mathcal{R}_{T}^{u}\right|
\end{array} \quad \text { where } k \in\{1,2, \ldots, n\}\right.
$$

Here, $\lambda_{-}^{T}$ is the shrink rate initialised by the user in the $T^{t h}$ interaction and ranges between $0<\lambda_{-}^{T} \leq 1$. When $\lambda_{-}^{T}$ is zero, the space shrinking process terminates. $\mathcal{R}_{T}^{l}$ and $\mathcal{R}_{T}^{u}$ are the continuous growth and decay parameters, respectively, which are computed using Equation 8 after the interaction $T$.

$$
\left\{\begin{array}{l}
x_{t, k}=x_{m, k}^{l} \times \exp \left(\mathcal{R}_{T}^{l} \times T\right) \\
x_{t, k}=x_{m, k}^{u} \times \exp \left(\mathcal{R}_{T}^{u} \times T\right)
\end{array}\right.
$$

Equation 8a represents the continuous exponential decay of the design space during the interactive process. $x_{t, k}$ represents the amount after shrinkage and $x_{m, k}^{l}$ is the initial amount at the $T^{\text {th }}$ interaction. Solving the Equation 8a for $R_{T}^{l}$ and $R_{T}^{u}$ yields Equation $8 \mathrm{~b}$.

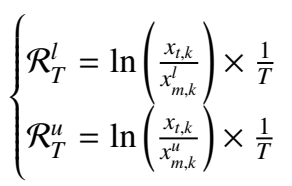

After obtaining the shrunk space, $x_{m, k}^{l}$ and $x_{m, k}^{u}$ are set equal to $\hat{x}_{m, k}^{l}$ and $\hat{x}_{m, k}^{u}$ respectively. Additionally, GenYacht has the ability to expand the design space. If the user is not satisfied with the designs in an interaction he/she can expand the design space instead of shrinking the space. During the expansion, the upper and lower bounds of $\mathcal{X}^{T}$ are constrained by the upper and lower bounds of the initial design space $\mathcal{X}$ (i.e., $\mathcal{X}^{T}:=\left\{\hat{x}_{t, k}^{l} \leq\right.$ $\left.\left.x_{t, k} \leq \hat{x}_{t, k}^{u}:\left(\hat{x}_{t, k}^{u} \leq x_{m, k}^{u}\right) \wedge\left(\hat{x}_{t, k}^{l} \geq x_{m, k}^{l}\right) \forall k \in\{1,2, \ldots n\}\right\}\right)$, which limits the new design space from over-expanding the initial design space. Expanding the design space is carried out using Equation 9.

$$
\left\{\begin{array}{l}
\hat{x}_{m, k}^{l}=x_{m, k}^{l}-\left|\lambda_{+}^{T} \times \mathcal{R}_{T}^{l}\right| \\
\hat{x}_{m, k}^{u}=x_{m, k}^{u}+\left|\lambda_{+}^{T} \times \mathcal{R}_{T}^{u}\right|
\end{array} \quad \text { where } k \in\{1,2, \ldots, n\}\right.
$$

Here, $\lambda_{+}^{T}$ is the expansion rate and ranges between $0<\lambda_{+}^{T} \leq$ 1. Algorithm 2 summarises the stepwise procedure of SST.

It should be noted that the parameter values should be scaled so that parameters with large values do not disproportionately affect the space shrinking or expanding process. Scaling is done using Equation 10 to avoid negative natural log values, where $[a, b]=[1,2]$.

$$
\mathbf{x}_{t} \mapsto \frac{\mathbf{x}_{t}-\mathbf{x}_{t}^{l}}{\mathbf{x}_{p_{t}}^{u}-\mathbf{x}_{t}^{l}} \times(b-a)+a
$$

To track the amount of shrinkage or expansion for the design space after each interaction, we introduce a quantity $Q$ (see Equation 11), which calculates the average percentage of shrinkage or expansion amount in the $T^{\text {th }}$ interaction for the dimensions of the design space.

$$
Q=\frac{1}{n} \times \sum_{k=1}^{n}\left(100-\frac{\hat{x}_{t, k}^{u}-\hat{x}_{t, k}^{l}}{x_{t, k}^{u}-x_{t, k}^{l}} \times 100\right)
$$

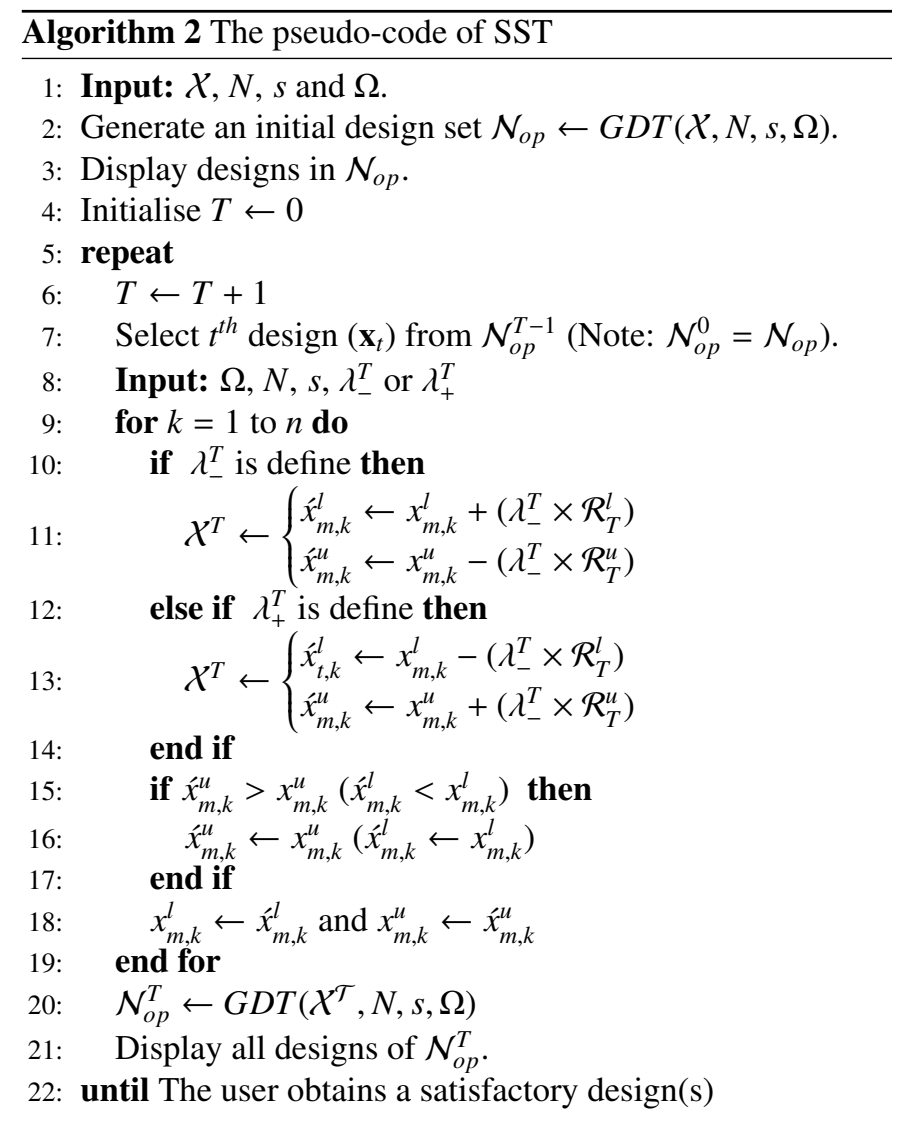

In an interaction between the user and GenYacht, the user can select multiple designs. Let the user select two designs, $\mathbf{x}_{t_{1}}$ and $\mathbf{x}_{t_{2}}$. Two design spaces, $\mathcal{X}_{1}^{T}$ and $\mathcal{X}_{2}^{T}$, are then formed. Therefore, two solution sets, $\mathcal{N}_{o p 1}^{T}$ and $\mathcal{N}_{o p 2}^{T}$, are separately obtained using 
GDT so that $2 \times N$ designs are shown to the user in the next interaction $(T+1)$.

In the $(T+1)^{\text {th }}$ interaction, if the user again selects two designs, one from $\mathcal{N}_{o p 1}^{T}$ and other from $\mathcal{N}_{o p 2}^{T}$, then again $2 \times N$ designs are created. However, if the user selects a design from $\mathcal{N}_{o p 1}^{T}\left(\mathcal{N}_{o p 2}^{T}\right)$, then $\mathcal{N}_{o p 2}^{T}\left(\mathcal{N}_{o p 1}^{T}\right)$ is discarded and $\mathcal{X}_{1}^{T+1}\left(\mathcal{X}_{2}^{T+1}\right)$ is formed to create $N$ designs for further interactions. If the user selects two designs from $\mathcal{N}_{o p 1}^{T}\left(\mathcal{N}_{o p 2}^{T}\right), 2 \times N$ designs are generated and $\mathcal{N}_{o p 2}^{T}\left(\mathcal{N}_{o p 1}^{T}\right)$ is discarded.

\subsection{User-Interface of GenYacht}

GenYacht is programmed in a Microsoft Visual Studio platform using the $\mathrm{C}++$ programming language and Parasolid's (a 3D geometric modelling kernel) API functions. A parent hull, shown in Figure 3, is initially stored in the database. During the interactive process, design modification of parent shape is performed using parametric design approach proposed by Khan et al. [22]. In this design framework, the overall hull shape is divided into three regions: Entrance-Region (ER), MiddleRegion (MR) and Run-Region (RR). Each region is then represented with a set of geometric parameters such as length $(L)$, beam $(B)$, depth $(D)$. Moreover, the entrance region is further constituted of three more geometric parameters: entrance angle $(\theta)$, bow angle $(\beta)$ and sheer angle $(\alpha)$. The parametric representation of the parent hull can be seen in Figure 3. The description of these parameters with their upper and lower bounds values (in meters) used for the study's experiments are given in Table 2 .

The main window of GenYacht consists of an OpenGL based graphical interface for design visualisation (see Figure 4). There are several dialog boxes in GenYacht for interactive designs, for calculating hydrostatics and resistance, and for setting the initial design space. To start the interactive design, the user first retrieves the parent hull using the 'initial design button' in the main window. The user then inputs the number of designs to be generated in the interactive design dialog box and selects the geometric parameters. To create a design space that is used in the interactive design process, the user can set any values for the upper and lower bounds of geometric parameters using the design space dialog box. Gen Yacht generates the specified number of yacht hulls, and the physical results of these hulls can be calculated at a user given draft value and Froude number. Based on the designs' form appearance and physical results, the user next makes design selection(s). Along with the selected design(s), the user inputs the shrink/expand rate value in the interactive design dialog box, which generates designs in the shrunk/expanded space for the next interaction. The user keeps interacting with GenYacht until the desired number of final designs are obtained.

GenYacht also provides users with the ability to define different geometric constraints any time during the interactive process(such as overall length $\left(L_{O A}\right)$, maximum beam $\left(B_{\max }\right)$ and maximum depth $\left.\left(D_{\max }\right)\right)$. The physical constraints can also be implemented in GenYacht to generate a hull with specific performance characteristics. For instance, a user can put a constraint to create designs with specific resistance value. However, care should be taken while defining the constraints and design space, as there can be a case when hull with a particular performance criterion might not be generated within the given design space. GenYacht notifies the user on the occurrence of such a situation. A user can also export the final design in the .x_t file format, which can be later imported to other digital platforms for further design and performance analysis. Hydrostatics and resistance results of the hull design can also be exported to a .xlsx file for future study.

\section{Results and Discussion}

In this section, we first compare the performance of five different meta-heuristics while integrating them with GDT. Afterwards, the efficiency of the GDT and SST is demonstrated with various experiments, and the effectiveness of GenYacht is also validated with a user study. Finally, we compared the performance of GenYacht with IGA.

\subsection{Optimiser Selection for GDT}

Five different optimisation techniques, GA, PSO, ABC, TLBO and JA, were tested in this section. Among them, TLBO and JA are newly proposed yet powerful techniques, which does not require any parameters to tune their convergence performance. Therefore, this quality of TLBO and JA alleviate an extra burden from the user during the design process. Our aim of testing these optimisation techniques was to select the one having converged to the least value of $\mathbf{F}(\mathcal{N})$ (Equation 6) in lesser computational time. As mentioned in [19], an interactive design approach with high computation cost may result in user fatigue and longer waiting time between interaction loops can cause loss of user interest.

Mutation and crossover rates were, respectively, set to 0.1 and 0.8 in GA, which control the exploration and exploitation of the search process. The linear-decreasing-inertia-weight was used and cognitive $\left(c_{1}\right)$ and social $\left(c_{2}\right)$ learning factors were taken as 2 in PSO. The number of employed and onlooker bees were set to the size of the sub-population $(s)$ in ABC. The performances of these optimisation techniques were tested under the standard algorithmic settings of $N=10, n=12, s=10$ and $\Omega=6$. Figure 5 shows a plot between $\mathbf{F}(\mathcal{N})$ and number of iterations. The computational time taken by the optimisers is given in Table 3.

It can be observed from the plot in Figure 5 that JA, GA and TLBO have similar performance, while JA converged to a lower value of $\mathbf{F}(\mathcal{N})$, and it can create completely non-collapsing designs (see Table 3). Based on these results, JA was selected to be used in GDT to update the designs in the sub-populations.

\subsection{Validation of GenYacht System}

In this section, the results of GDT and SST will be given, which are embedded in the Gen Yacht system.

\subsubsection{Results of GDT}

Figure 6 shows 20 space-filling design alternatives for the parent hull, which were generated using GDT. These alternatives are searched within a 12-dimensional design space bounded with parametric limits shown in Table 2 and with the 


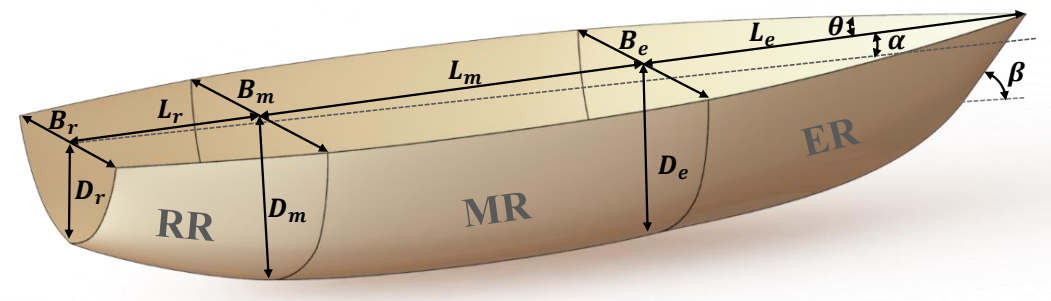

Figure 3: Parametric representation of the parent yacht hull created using Khan et al.'s design technique [22]. The parent hull is divided into three regions: Entrance, Middle and Run. Each region is represented with independent set of geometric parameters.

Table 2: Geometric parameters with their lower and upper bounds for the yacht hull.

\begin{tabular}{|c|c|c|c|c|c|}
\hline Parameters & Definition & {$[\mathrm{LB}, \mathrm{UB}]($ meters) } & Parameter & Definition & {$[\mathrm{LB}, \mathrm{UB}](\mathrm{meters})$} \\
\hline$L_{e}$ & Length of ER & {$[5.0,8.0]$} & $D_{e}$ & Depth of ER & {$[2.3,4.3]$} \\
\hline$L_{m}$ & Length of MR & {$[4.0,10.0]$} & $D_{m}$ & Depth of MR & {$[2.2,4.2]$} \\
\hline$L_{r}$ & Length of RR & {$[2.0,6.0]$} & $D_{r}$ & Depth of RR & {$[1.7,3.0]$} \\
\hline$B_{e}$ & Beam of ER & {$[5.0,7.0]$} & $\theta$ & Entrance Angle & {$\left[30^{\circ}, 90^{\circ}\right]$} \\
\hline$B_{m}$ & Beam of MR & {$[5.4,7.4]$} & $\beta$ & Bow Angle & {$\left[30^{\circ}, 100^{\circ}\right]$} \\
\hline$B_{r}$ & Beam of RR & {$[3.4,5.4]$} & $\alpha$ & Sheer Angle & {$\left[0^{\circ}, 3^{\circ}\right]$} \\
\hline
\end{tabular}

where LB: Lower Bound, UB: Upper Bound

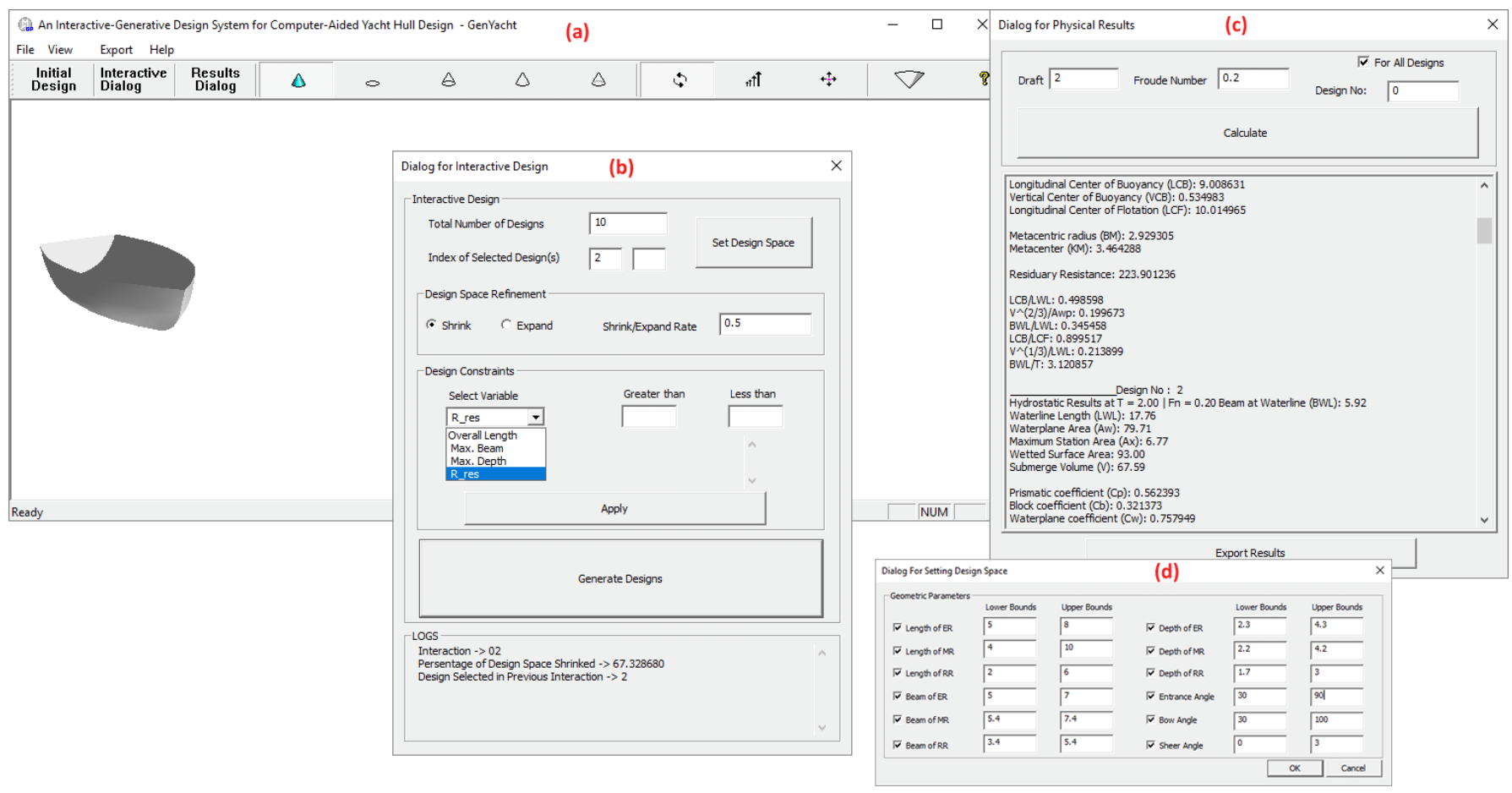

Figure 4: The user interface of GenYacht consists of a main window (a), a dialog box for user-GenYacht interaction (b), a dialog box for calculating hydrostatics and resistance (c) and a dialog box for setting the initial design space (d).

Table 3: Computational times for GA, PSO, ABC, TLBO and JA when used with Algorithm 1

\begin{tabular}{|c|c|c|c|}
\hline & Computational Time (minutes) & Space-filling $\left(F_{1}\right)$ & Collapsing Designs \\
\hline JA & $\mathbf{0 . 8 4}$ & $\mathbf{1 6 . 5 6}$ & $\mathbf{0}$ \\
\hline TLBO & 3.51 & 17.82 & 3 \\
\hline GA & 2.14 & 17.95 & $\mathbf{0}$ \\
\hline ABC & 1.40 & 18.79 & 3 \\
\hline PSO & 2.94 & 19.06 & 6 \\
\hline
\end{tabular}




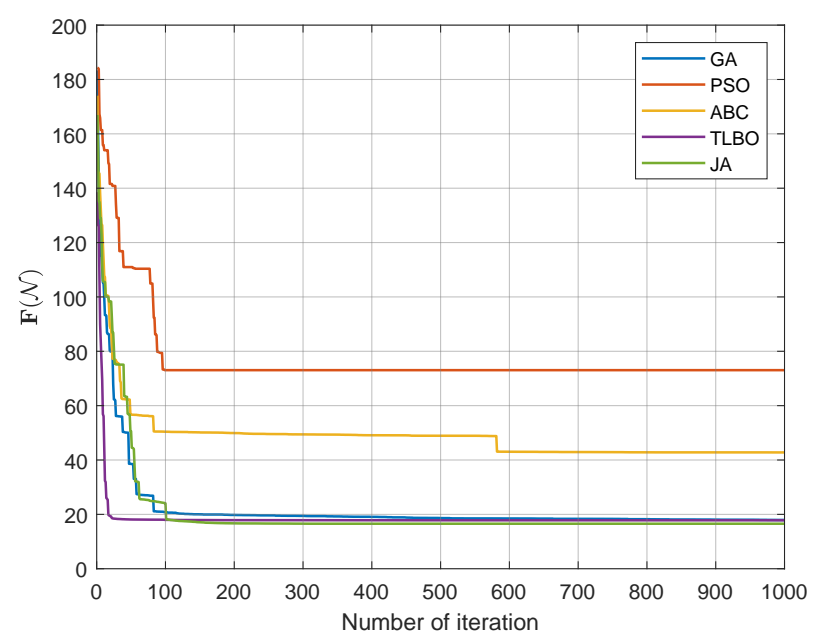

Figure 5: Plots for the objective function $(F(\mathcal{N}))$ versus number of iterations performed in GA, PSO, ABC, TLBO and JA.

parameter settings of $s=10$ and $\Omega=6$. From Figure 6, one can easily observe that the designs are distinct from each other, which can help users of GenYacht to start the interactive process with a design that meets his/her requirements. As mentioned before, the design modification is performed using Khan et al.'s parametric design framework [22], which locally modifies the geometric parameters. To ensure the generation of plausible shapes (i.e., realistic hull shapes) in interactions, following hard design constraints have been implemented: (1) $-\frac{B_{e}}{3} \leq\left(B_{e}-B_{m}\right)$, (2) $-\frac{D_{e}}{3} \leq\left(D_{e}-D_{m}\right)$, (3) $B_{r} \leq\left(B_{m}, B_{e}\right)$ and (4) $D_{r} \leq\left(D_{m}, D_{e}\right)$. The first two constraints limits the parameters $B_{m}$ and $D_{m}$, and last two constrains $B_{r}$ and $D_{r}$. According to our experience, if $B_{m}>>B_{e}$ or $D_{m}>>D_{e}$, and if $B_{r}>>\left(B_{m}, B_{e}\right)$ or $D_{r} \gg>\left(D_{m}, D_{e}\right)$, implausible designs can occur as shown in Figure 7 (a) and (b), respectively.

During the hull form design, there are a variety of numerical performance analyses, consisting of both hydrostatics and hydrodynamics, that naval engineers have to perform to determine whether the hull form can fulfil the design requirements before the selection of final design. Therefore, using Gen Yacht, users can also evaluate the hydrostatics properties, form coefficients, residuary $\left(R_{\text {res }}\right)$ and frictional $\left(R_{F}\right)$ resistance of each hull design at the given values of the $\operatorname{draft}(T)$ and Froude numbers $\left(F_{n}\right)$. Table 4 shows the hydrostatics and resistance results for the first ten designs in Figure 6. It should be noted that the hydrodynamics of a hull includes wave resistance, sea-keeping, manoeuvrability, and so forth, which mostly require Computational Fluid Dynamic (CFD) analyses to be performed. However, running these computationally expensive analyses make the user wait for a long time before performing the next interaction. This can also result in directing the exploration process towards the non-preferred regions. Therefore, we have utilised empirical equations, proposed by Keuning and Katgert [57], to calculate the $R_{\text {res }}$ of the hull alternatives. Figure 8 shows the plots of $R_{\text {res }}$ (expressed in Newton) versus $F_{n}$ of the first six designs in Figure 6. The differences of the appearances and performances for the designs in the plots of Figure 8 demon- strate that the designs generated by the proposed system in Figure 6 are diverse in terms of both appearance and performance. Figure 8 also validates the implementation of Keuning and Katgert's [57] technique to calculate residuary resistance at different Froude numbers. Frictional resistance is also calculated according to the ITTC formula [58]. Reynolds number $\left(R_{n}\right)$ and frictional resistance coefficient $\left(C_{F}\right)$ are calculated for a yacht navigating in seawater at $15^{\circ} \mathrm{C}$ with density and kinematic viscosity of $1.189 \times 10^{-6}\left(\mathrm{~m}^{2} / \mathrm{s}\right)$ and $1026.021\left(\mathrm{~kg} / \mathrm{m}^{3}\right)$, respectively. The total resistance $\left(R_{T}\right)$ is the sum of $R_{r e s}$ and $R_{F}$.

It should be noted that the core objective of this work is to propose an interactive design system, which gives users the ability to generate yacht hull designs at the preliminary stage while taking its form appearance and physical properties into account. After selecting the desired hull form(s), the user can export it and then can perform detailed hydrodynamic and structural analyses using off-the-shelf computational tools.

\subsubsection{Results of SST}

The results of SST were validated with different experiments using different values of the shrink rate $\left(\lambda_{-}\right)$. Ten design alternatives were first generated, and interaction then proceeded with an objective to select a design having a trade-off between appearance and performance. Figure 9 (a), (b) and (c) shows the designs created in the fifteen design interactions with $\lambda_{-}$ settings of 0.1, 0.5 and 1.0, respectively. Figure 10 (a), (b) and (c) shows plots for the average percentage of space-shrinkage $Q$ versus the design interactions ( $T$ ) in Figure 9 (a), (b) and (c), respectively. In Figure 10, the top axis (in red colour) shows the design selected in each interaction for design in 9. The area under the curve represents the percentage of the space shrunk in fifteen interactions. It is noteworthy that at higher values of $\lambda_{-}$, the value of $Q$ is high in the interactions. For instance, when $\lambda_{-}$was set to $0.1,0.5$ and 1.0 , the original design space shrunk by $6.93 \%, 34.66 \%$ and $69.31 \%$ in the first interaction $(T=1)$, respectively. Afterwards, in the second interaction $(T=2), 3.47 \%, 17.32 \%$, and $34.66 \%$ per cent of the design space created in the first interaction was shrunk.

As mentioned before, at each interaction user selects a design and depending on the value of shrink/expend rate design space is shrunk/expended and new $N$ designs are generated for the next interaction. At higher values of $\lambda_{-}$, the amount of design space shrinks is higher (see Figure 10 (c)), which might create a narrower design space. The designs generated from this space for the next interaction can be similar (i.e. designs with less diverse, see designs in Figure 9 (c)). When $\lambda_{-}$is set to higher values during interactions, the designs converge faster (i.e., get similar) towards the selected design. For instance, interaction results, which are shown in Figure 9 (c) were obtained using $\lambda_{-}=1.0$. In this setting, designs started to converge after the third interaction.

On the contrary, designs generated from a design space created with a smaller value of $\lambda_{-}$will be more diverse. As shown in Figure 10 (a), when $\lambda_{-}$was set equal to a very small value (i.e. $\left.\lambda_{-}=0.1\right)$, the shrinkage of the design space in each interaction is small and designs generated are diverse. Thus, it may require a higher number of iterations for a user to converge to 

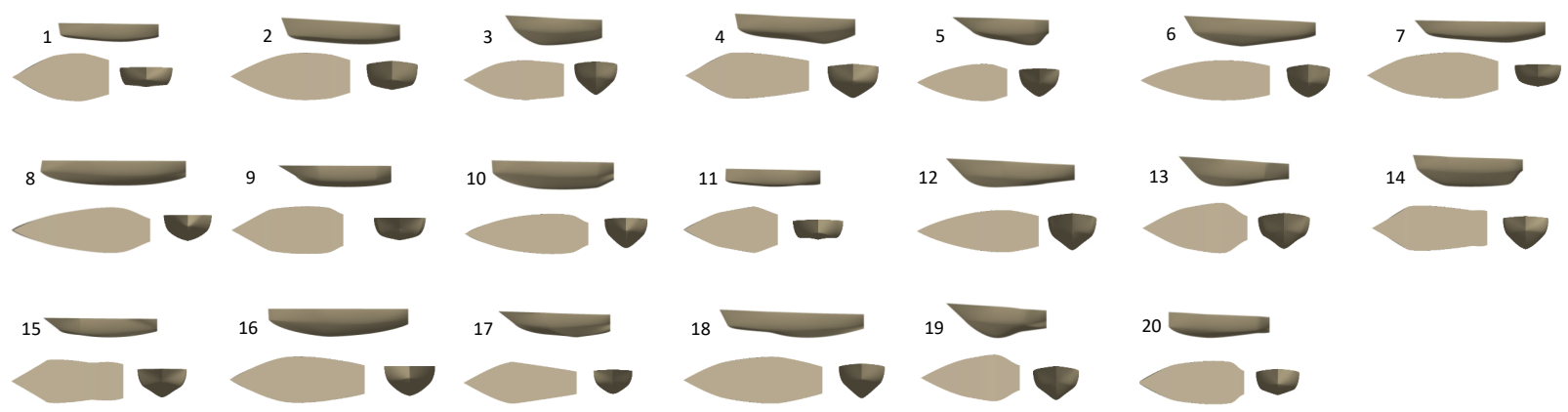

Figure 6: Design alternatives generated using GDT for the hull model in Figure 3 (For better visualisation of designs in this figure, the reader is referred to the digital version of this article).

Table 4: Hydrostatics and resistance results of the first ten design alternatives shown in Figure 6.

\begin{tabular}{|c|c|c|c|c|c|c|c|c|c|c|}
\hline Hull & 1 & 2 & 3 & 4 & 5 & 6 & 7 & 8 & 9 & 10 \\
\hline$D$ & 2.54 & 2.95 & 4.00 & 3.99 & 3.61 & 3.68 & 3.57 & 3.18 & 4.02 & 2.50 \\
\hline$T$ & 1.00 & 1.00 & 1.50 & 1.50 & 1.50 & 1.50 & 1.50 & 1.50 & 1.50 & 1.50 \\
\hline$F_{n}$ & 0.20 & 0.20 & 0.20 & 0.20 & 0.20 & 0.20 & 0.20 & 0.20 & 0.20 & 0.20 \\
\hline$B W L$ & 6.83 & 6.35 & 4.11 & 5.25 & 4.37 & 4.87 & 5.79 & 6.70 & 4.28 & 6.47 \\
\hline$L W L$ & 14.78 & 16.96 & 13.04 & 16.96 & 10.08 & 17.33 & 21.04 & 14.59 & 17.58 & 14.79 \\
\hline$A_{w p}$ & 70.31 & 75.92 & 36.96 & 60.14 & 31.95 & 58.90 & 78.29 & 77.61 & 48.61 & 62.72 \\
\hline$A_{x}$ & 5.18 & 4.15 & 3.43 & 3.93 & 3.81 & 4.22 & 5.42 & 7.15 & 3.80 & 4.22 \\
\hline$A_{w s}$ & 77.38 & 80.31 & 46.64 & 67.67 & 37.28 & 69.03 & 92.56 & 88.64 & 63.60 & 69.98 \\
\hline$V$ & 42.31 & 38.90 & 26.46 & 33.60 & 20.26 & 37.06 & 63.10 & 72.09 & 37.75 & 36.75 \\
\hline$I T$ & 200.35 & 194.14 & 36.03 & 92.48 & 37.10 & 84.76 & 147.99 & 233.29 & 52.26 & 145.05 \\
\hline$L C B$ & 8.41 & 10.09 & 6.36 & 9.45 & 6.25 & 9.05 & 11.70 & 7.97 & 10.10 & 8.55 \\
\hline$K B$ & 0.34 & 0.32 & 0.48 & 0.40 & 0.44 & 0.44 & 0.50 & 0.54 & 0.51 & 0.32 \\
\hline$L C F$ & 8.30 & 9.44 & 6.66 & 8.52 & 5.82 & 10.03 & 11.72 & 7.87 & 9.98 & 8.44 \\
\hline$B M$ & 4.74 & 4.99 & 1.36 & 2.75 & 1.82 & 2.29 & 2.35 & 3.24 & 1.38 & 3.95 \\
\hline$K M$ & 5.07 & 5.30 & 1.84 & 3.15 & 2.67 & 2.72 & 2.85 & 3.77 & 1.90 & 4.27 \\
\hline$C_{p}$ & 0.55 & 0.55 & 0.59 & 0.50 & 0.53 & 0.51 & 0.55 & 0.69 & 0.56 & 0.59 \\
\hline$C_{b}$ & 0.42 & 0.36 & 0.33 & 0.25 & 0.31 & 0.29 & 0.35 & 0.49 & 0.33 & 0.40 \\
\hline$C_{w p}$ & 0.70 & 0.70 & 0.69 & 0.68 & 0.73 & 0.70 & 0.64 & 0.79 & 0.65 & 0.66 \\
\hline$C_{m}$ & 0.76 & 0.65 & 0.56 & 0.50 & 0.58 & 0.58 & 0.62 & 0.71 & 0.59 & 0.69 \\
\hline$R_{r e s}$ & 353.10 & 251.74 & 83.01 & 145.27 & 166.24 & 114.05 & 225.99 & 417.82 & 121.06 & 282.74 \\
\hline$R_{F}$ & 575.80 & 663.84 & 315.53 & 559.36 & 207.67 & 580.12 & 903.01 & 653.12 & 310.98 & 521.01 \\
\hline$R_{T}$ & 928.90 & 915.58 & 398.54 & 704.63 & 373.91 & 694.17 & 1129 & 1070.94 & 432.04 & 803.75 \\
\hline
\end{tabular}

where $D$ : Depth $(m), T$ : Draft $(m), B W L$ : Width at waterline $(m), L W L$ : Length at waterline $(m), A_{w}$ : Waterplane area $\left(m^{2}\right), A_{x}$ : Maximum sectional area $\left(m^{2}\right)$, $A_{w s}$ : Wetted surface area $\left(\mathrm{m}^{2}\right), V$ : Volume $\left(\mathrm{m}^{3}\right), I T$ : Transverse moment of inertia $\left(\mathrm{m}^{4}\right), L C B$ : Longitudinal center of buoyancy $(m), K B$ : Vertical center of buoyancy $(m), L C F$ : Longitudinal center of flotation $(m), B M$ : Metacentric radius $(m), K M$ : Metacenter height $(m), C_{p}$ : Prismatic coefficient, $C_{b}$ : Block coefficient, $C_{w p}$ : Waterplane coefficient, $C_{m}$ : Midship coefficient, $R_{r e s}$ : Residuary Resistance $(N), R_{F}$ : Frictional Resistance $(N)$ and $R_{T}$ : Total Resistance $(N)$.

the final design. However, for small values of $\lambda_{-}$, the user can explore more variety of designs. For instance, design generated in Figure 9 (a) are created when $\lambda_{-}=0.1$, therefore, even at the $15^{\text {th }}$ interaction, designs are still diverse, thereby showing a slow convergence. Moreover, as shown in Figure 9 (b), at $\lambda_{-}=0.5$, the user can achieve a better trade-off between design diversity and convergence because the amount of space shrinks at each interaction is moderate (see Figure 10 (b), which shows the amount of space shrinks at each interaction when $\lambda_{-}=0.5$ ). Therefore, we recommend the users to start the interaction at $\lambda_{-}=0.5$. Figure 11 shows a plot between $Q$ and $\lambda_{-}$, which confirms a linear relationship between these two parameters.
The ability of the proposed system to search for a target design was also tested. First, a target design was randomly selected from the design space in Table 2 and its parameter values and hydrostatic properties were stored. Afterwards, the interactive process was started with an aim to replicate the target design. At the first interaction, 20 designs were generated and from these designs, a design having parameter and hydrostatics values close to the target one is selected. Based on the selected one, 20 new designs were generated and the process was repeated for four interactions. The target and final design obtained after the fourth interaction is shown in Figure 12. It can be observed that visually both designs are very similar, more- 


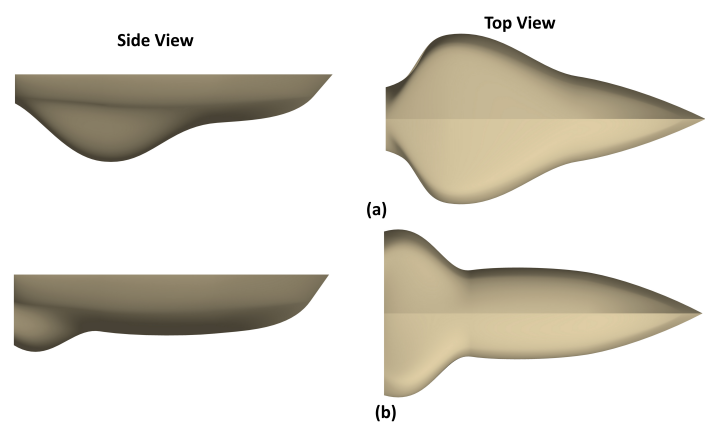

Figure 7: Example of implausible/non-realistic designs in the absence of hard design constraints.
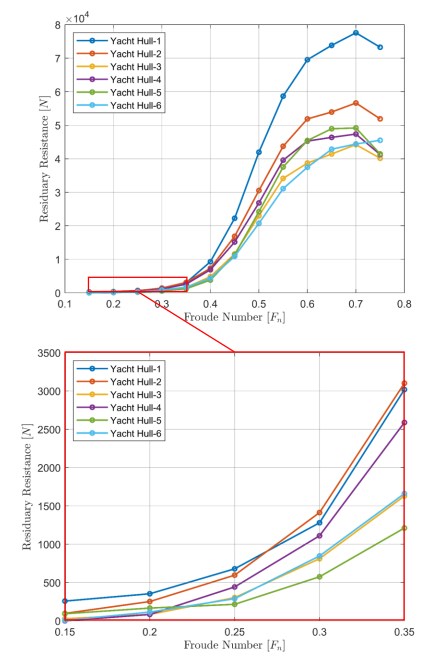

Figure 8: Plot showing the residuary resistance $\left(R_{\text {res }}\right)$ versus froude number $\left(F_{n}\right)$ for the first six designs in Figure 6.

over, their parameter values and hydrostatic properties, which are shown in Table 5, are also close to each other. This validates the ability of GDT and SST technique to converge to the desired hull design.

Here, it is noteworthy that the interactive process should be started with an appropriate number of designs to visualize all the uniformly-distributed designs that sufficiently covers the design space. However, this number can be high particularly for the high-dimensional design spaces or the design spaces whose dimensional bounds are large. In such cases, the claim that the proposed method removes the user fatigue may not hold true. However, as proven via experiments, using GenYacht the user can still explore a design space well compare to IGA.

\subsection{Computational Time}

As mentioned before, one of the crucial criteria in interactive design techniques is that it should be computationally less expensive. As longer waiting times cause user fatigue, thereby hindering the user from effectively exploring the design space for a satisfactory design. The experiments in this study were conducted using a $\mathrm{PC}$ with an i7-7700 Intel Core, 3.6-GHz processor, and 8-GB physical memory. Figure 13 shows a plot of the computational time (in seconds) of GenYacht versus the number of designs $(N)$ generated. The computational cost is the sum of the computational time taken by GDT to explore $N$
Table 5: Parametric values and hydrostatic properties of the design shown in Figure 12. Hydrostatic properties were calculated at the draft of 2.0 meters.

\begin{tabular}{|c|c|c|}
\hline Parameters (units) & Target Design & Generated Design \\
\hline$L O A(m)$ & 20.72 & 20.07 \\
\hline$B_{\max }(m)$ & 6.62 & 6.84 \\
\hline$D_{\max }(m)$ & 3.49 & 3.71 \\
\hline$A_{w p}\left(m^{2}\right)$ & 92.26 & 92.69 \\
\hline$A_{x}\left(m^{2}\right)$ & 7.89 & 7.38 \\
\hline$A_{w s}\left(m^{2}\right)$ & 116.23 & 113.12 \\
\hline$V\left(m^{3}\right)$ & & 96.06 \\
\hline$I T\left(m^{4}\right)$ & 205.70 & 193.46 \\
\hline$L C B(m)$ & 11.20 & 11.17 \\
\hline$K B(m)$ & 0.670 & 0.645 \\
\hline$L C F(m)$ & 11.40 & 11.56 \\
\hline$B M(m)$ & 1.98 & 2.05 \\
\hline$K M(m)$ & 2.65 & 2.70 \\
\hline$C_{p}$ & 0.636 & 0.608 \\
\hline$C_{b}$ & 0.414 & 0.382 \\
\hline$C_{w p}$ & 0.737 & 0.754 \\
\hline$C_{m}$ & 0.651 & 0.629 \\
\hline
\end{tabular}

space-filling designs, parametric modification of $N$ designs using Khan et al.'s [22] approach, computation of hulls' physical properties and space-refinement in one interaction. From Figure 13, it can be seen that GenYacht took approximately two minutes to create 50 designs in an interaction. These results confirm that the computational complexity of the proposed system is significantly low.

\subsection{User Study}

A user study was conducted to validate the efficiency and feasibility of GenYacht. We selected ten PhD candidates as the subjects in the user study from the Department of Naval Architecture, Ocean and Marine Engineering at the University of Strathclyde, who had an average 3.60 \pm 2.67 (average \pm standard deviation) years of industrial and research experience in the ship and parametric design. A brief introduction of interactive designs approach was first presented to the subjects, and a small training session of the proposed system was then given along with a description of SST and its behaviour with the shrink rate. Plots in Figure 10 and 11 were described to them, so they can have a better understanding of tuning this parameter. Subjects were also familiarised with geometric parameters of the primary hull form, and they were asked to set some design specifications before starting the interactive process. To avoid user fatigue, we asked subjects first choose some designs based on their form appearance and then compare these designs based on the physical performance before making the final selection or vice versa. The results of the interactive process for each subject are shown in Table 6, and the designs generated by the subjects are shown in Figure 14. The average time taken by the subjects to complete the interactive process was $5.12 \pm 1.07$ minutes.

After the interactive process was completed, we asked the 


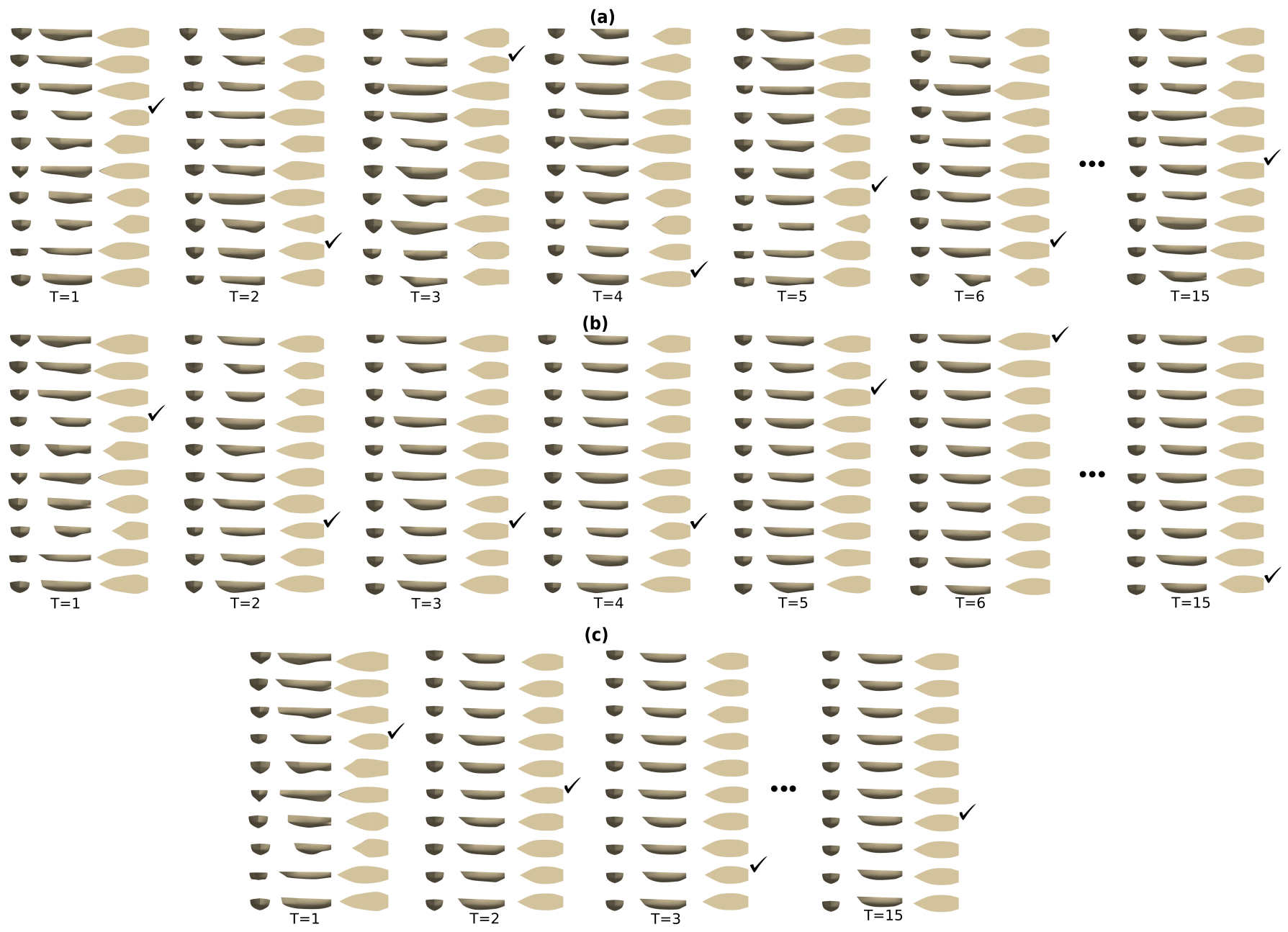

Figure 9: Yacht hull alternatives created during fifteen design interactions using a shrink rate ( $\lambda_{-}$) of (a) 0.1 , (b) 0.5 and (c) 1.0 (For better visualisation of designs in this figure, the reader is referred to the digital version of this article).
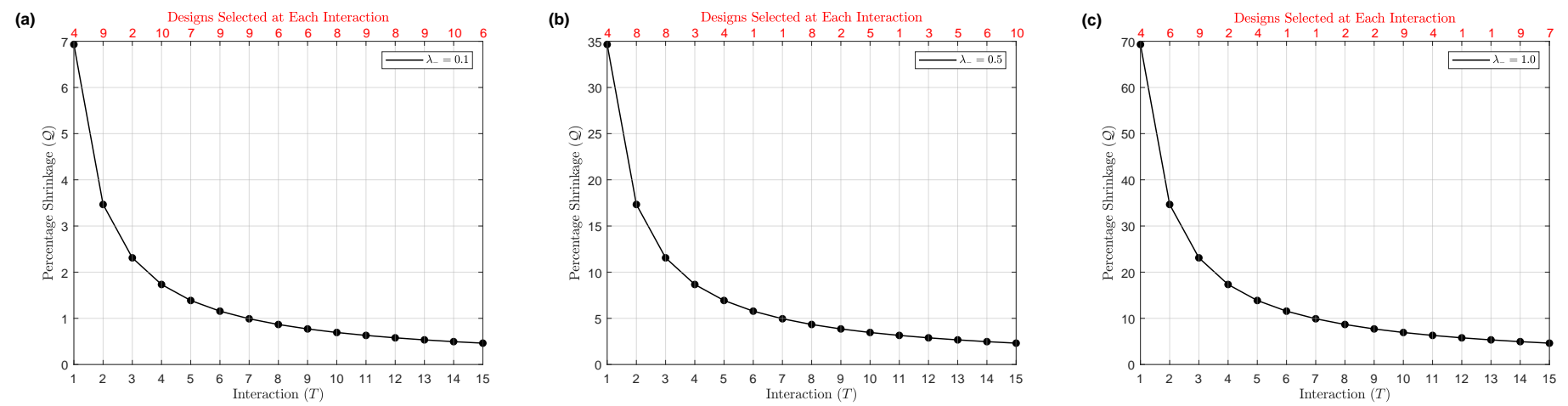

Figure 10: Plots showing the percentage shrinkage $(Q)$ of the design space during fifteen design interactions $(T)$ when (a) $\lambda_{-}=0.1$, (b) $\lambda_{-}=0.5$ and $\lambda_{-}=1.0$.

following questions to the subjects for further evaluation of the system. Their responses were acquired on a 5-point Likert scale (1: Strongly Disagree, 2: Disagree, 3: Neutral, 4: Agree, 5: Strongly Agree):

Q1: Gen Yacht is easy to use in an interactive generation of hull forms.

Q2: GenYacht yacht provides a more sophisticated approach for preliminary hull design compared to the traditional parametric design exploration techniques.

Q3: Using GenYacht, I was able to generate a satisfactory design within my design requirements.

The average of the Likert scores given by the subjects

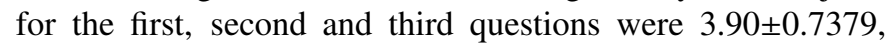
$4.40 \pm 0.6992$ and $4.00 \pm 0.9428$, respectively. The variations of 
Table 6: Results of the user study.

\begin{tabular}{|c|c|c|c|c|c|c|c|c|c|c|c|}
\hline Interaction & Subjects & 1 & 2 & 3 & 4 & 5 & 6 & 7 & 8 & 9 & 10 \\
\hline & Initial Designs $(N)$ & 15 & 25 & 17 & 10 & 10 & 25 & 21 & 17 & 15 & 20 \\
\hline \multirow{3}{*}{$T=1$} & Design Selected & 8 & 24,7 & 3 & 1 & 9 & 20 & 9 & 15 & 8 & 9 \\
& $\lambda_{-}^{1}$ & 0.5 & 0.5 & 0.5 & 0.5 & 0.5 & 0.5 & 0.5 & 0.3 & 0.7 & 0.5 \\
& $N^{1}$ & 10 & 10 & 17 & 13 & 10 & 5 & 21 & 17 & 15 & 20 \\
\hline \multirow{3}{*}{$T=2$} & Design Selected & 7 & 19 & 1 & 4 & 8 & 1 & 15 & 2 & 10 & 6 \\
& $\lambda_{-}^{2}$ & 0.5 & 0.5 & 0.5 & 0.8 & 0.5 & 0.5 & 0.8 & 0.5 & 0.8 & 0.3 \\
& $N^{2}$ & 7 & 10 & 10 & 6 & 10 & 10 & 21 & 17 & 15 & 20 \\
\hline \multirow{3}{*}{$T=3$} & Design Selected & 3 & 1 & 4 & 1,2 & 5 & 1 & 8 & 15 & $\mathbf{9}$ & 12 \\
& $\lambda_{-}^{3}$ & 0.5 & 0.5 & 0.5 & 0.8 & 0.7 & 0.9 & 1.0 & 0.6 & - & 0.3 \\
& $N^{3}$ & 5 & 5 & 5 & 3 & 10 & 5 & 21 & 20 & - & 15 \\
\hline \multirow{4}{*}{$T=4$} & Design Selected & 3 & 2 & 1 & 3 & 6 & $\mathbf{1}$ & $\mathbf{1 5}$ & 9 & - & $\mathbf{1 3}$ \\
& $\lambda_{-}^{4}$ & 0.5 & 0.5 & 1.0 & 1.0 & 0.9 & - & - & 0.6 & - & - \\
& $N^{4}$ & 5 & 5 & 5 & 6 & 20 & - & - & 20 & - & - \\
\hline \multirow{3}{*}{$T=5$} & Design Selected & $\mathbf{2}$ & $\mathbf{2}$ & 4 & $\mathbf{4}$ & 7 & - & - & 2 & - & - \\
& $\lambda_{-}^{5}$ & - & - & 1.0 & - & 0.9 & - & - & 0.8 & - & - \\
& $N^{5}$ & - & - & 3 & - & 5 & - & - & 20 & - & - \\
\hline \multirow{3}{*}{$T=6$} & Design Selected & - & - & $\mathbf{3}$ & - & $\mathbf{1}$ & - & - & 2 & - & - \\
& $\lambda_{-}^{6}$ & - & - & - & - & - & - & - & 1.0 & - & - \\
& $N^{6}$ & - & - & - & - & - & - & - & 20 & - & - \\
\hline$T=7$ & Design Selected & - & - & - & - & - & - & - & $\mathbf{3}$ & - & - \\
\hline
\end{tabular}

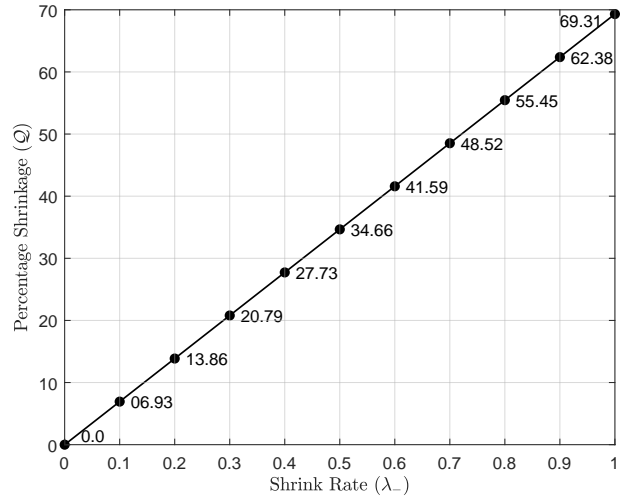

Figure 11: Plot showing the percentage shrinkage $(Q)$ of the design space versus shrink rate $\left(\lambda_{-}\right)$

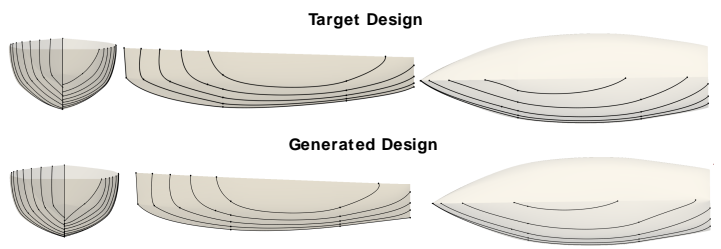

Figure 12: The design space is explored interactively to replicate a target design. The image at the top is the target design and the image at the bottom is the design generated after four interactions using GenYacht. The similarity between the two designs indicates that the user could well approximate the target design.

the user scores were also analysed using the Box and Whisker plot, which is shown in Figure 15. These results indicate that users could generate satisfactory designs using GenYacht.

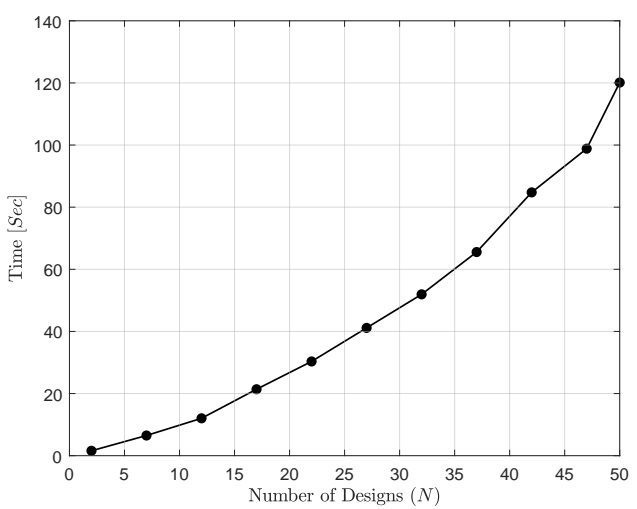

Figure 13: Plot showing GenYacht's computational cost (in seconds) versus number of designs $(N)$.

Some subjects also suggested that they would like to create parent shape using GenYacht, which they would like to further optimise for the specific performance criteria. Subjects also like that GenYacht gives users the ability to compare a wide variety of designs, which is essential in ship design because mostly the optimal configuration is the one that best satisfies the customers' design requirements.

\subsection{Comparison with IGA}

We have also compared the performance of GenYacht with an IGA-based technique. As mentioned in Section 2.1, there are many variations of IGA in literature. In this work, we implemented IGA similar to [19] and utilised first a two-dimensional design space formed using the geometric parameters, $L_{e}$ and 


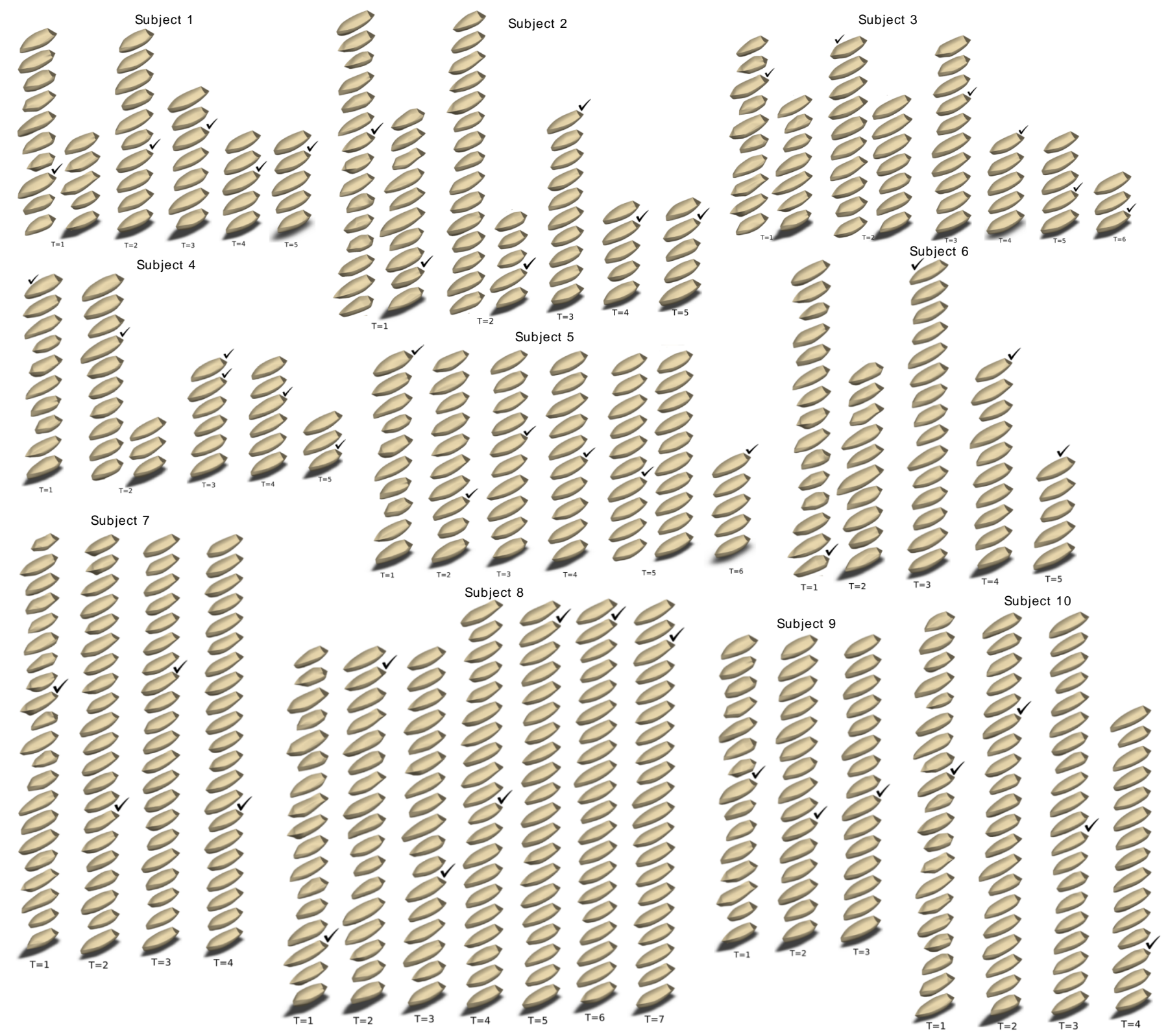

Figure 14: The designs generated by the subjects in the user study (For better visualisation of designs in this figure, the reader is referred to the digital version of this article).

$B_{e}$, of the parent hull for better visualisation of GenYacht's and IGA's performances (see Figures 16 and 18). However, we selected a preferred design, instead of rating the designs as this selection scenario is similar to GenYacht. An initial population of random designs was first generated, and a design was selected from the initial population. GA then performed an iteration/generation to create a new population with an objective to minimise the normalised Euclidian distance between the chosen design and designs in the population pool. In each generation, designs were evaluated to make a selection. Here, GA was used with a crossover and mutation rates of 0.8 and 0.1 , respectively. It can be seen from Figure 16 that after each generation of GA, the newly generated designs moved towards the selected design. Here, it is noteworthy that IGA focuses on the convergence towards the selected hull form instead of maintain- ing diversity. Furthermore, the main drawback of IGA is that it depends mainly on the initial population, therefore, starting the interactive process with randomly sampled designs may limit the exploration process. More than 50\% of the design space remained unexplored when IGA was used in Figure 16. Figure 17 highlights these unexplored regions.

Figure 18 (b) and (a) show the interactive results of GenYacht for a two-dimensional design space created with $L_{e}$ and $B_{e}$, and the shrunk design space at each interaction. It can be observed that, compared to IGA, GenYacht let the users start the design process with well-sampled diverse designs, and explores the design space effectively at each interaction. Moreover, SST provides a sophisticated way to focus the computational effort on the exploration of potential regions.

Figure 19 (a) shows the yacht hull designs generated using 


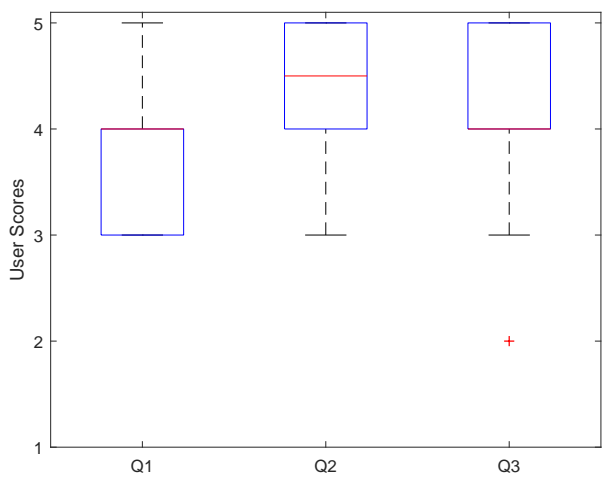

Figure 15: Box and Whisker plots of the scores given to question Q1, Q2 and Q3 given by the subjects during the user study. These questions were asked to the subjects at the end of user study for the further evaluations of GenYacht.

GDT and the left-most image of Figure 19 (b) shows the randomly sampled designs, which were then used to perform the interaction process using IGA. The space-filling values $\left(F_{1}(\mathcal{N})\right)$ of GDT and random designs is 17.2708 and 26.1553, respectively. The high value indicates that the random design does not spread in the design space evenly. A glance on the appearance of these designs can reveal that there exists a clustering pattern in randomly generated hull forms (first image of Figure 19 (b)). For instance, from the top; first three, next four and last three designs are similar. However, designs created via GDT (Figure 19 (a)) are unique to a large extent. Figure 19 (b) also shows the interactive results of IGA. It can be observed that the designs generated in each interaction are very similar as there is no control for the user maintaining the design diversity.

\section{Conclusions and Future Works}

This work proposes a novel interactive and generative design based CAD system for the preliminary design of yacht hull forms. The proposed system introduces a new design approach in the field of naval architecture, which enables naval architects, engineers and novice users to integrate their design preference about the hull form into the design space exploration. Users can generate designs which best fit their design requirements, not only in term of physical performance but also taking into account the design's overall appearance. In GenYacht, a generative design approach first generates a user-defined number of space-filling hull forms satisfying the given designs constraints. Among these designs, the user selects a suitable one, which is then used to create a new design space using a space-shrinking technique. The new space is then fed to the generative design technique to generate a set of space-filling designs for the next interaction. This generative and interactive process continues until the user reaches the desired shape. Experimental and user study results reveal that the proposed system has the potential to create user-centred yacht hull forms, which better reflects designers' design considerations. The new system also benefits the users in the field of naval architecture and marine engineering compared to the parametric based exploration techniques.
In future work, we plan to use non-dimensional parameters to define the hull design space and to test GenYacht with this design space. We would also like to integrate more physical performance criteria, such as sea-keeping and stability. Furthermore, we would like to develop empirical equations for these criteria using deep-learning. Our efforts will also continue to develop a web-based user-interface to give better usability to the potential users. Additionally, we think that it will be even worthy to work for the development of a similar interactive system for other types of marine vessels, such as chined hulls (or planing crafts) and multihulls.

\section{Acknowledgements}

We would like to pay our deepest gratitude to Professor Panagiotis Kaklis for his useful comments and help in implementing the empirical equations of residuary resistance. We would also like to thank $\mathrm{LibXL} \mathrm{TM}^{\mathrm{TM}}$ for providing free access to their $\mathrm{C}++\mathrm{li}$ braries, which were used to export physical results to .xlsx files and also the $\mathrm{PhD}$ candidates from HD216 Research Center at the Department of Naval Architecture, Ocean and Marine Engineering at the University of Strathclyde, who participated in the user study.

\section{References}

[1] K. M. Dogan, E. Gunpinar, Learning yacht hull adjectives and their relationship with hull surface geometry using gmdh-type neural networks for human oriented smart design, Ocean Engineering 145 (2017) 215-229.

[2] S. Khan, Development of a cad system for parametric and attribute-based modification of yacht hull models, Master's thesis, Istanbul Technical University (2017).

[3] T. P. McDonald, D. J. Andrews, R. G. Pawling, A demonstration of an advanced library based approach to the initial design exploration of different hullform configurations, Computer-Aided Design 44 (3) (2012) 209-223.

[4] R. Pawling, D. Andrews, Design sketching for computer aided preliminary ship design, Ship Technology Research 58 (3) (2011) 182-194.

[5] E. Duchateau, Interactive evolutionary concept exploration in preliminary ship design, Ph.D. thesis, Delft University of Technology (2016).

[6] B. J. Van Oers, A packing approach for the early stage design of service vessels, Ph.D. thesis, Delft University of Technology (2011).

[7] T. Van Bruinessen, H. Hopman, T. DeNucci, B. Van Oers, Generating more valid designs during design exploration, Journal of Ship Production and Design 27 (4) (2011) 153161.

[8] B. Hasubek, S. Harries, Simulation-driven design of sailing yachts and motor boats, in: Proceedings of the VII International Conference on Computational Methods in 

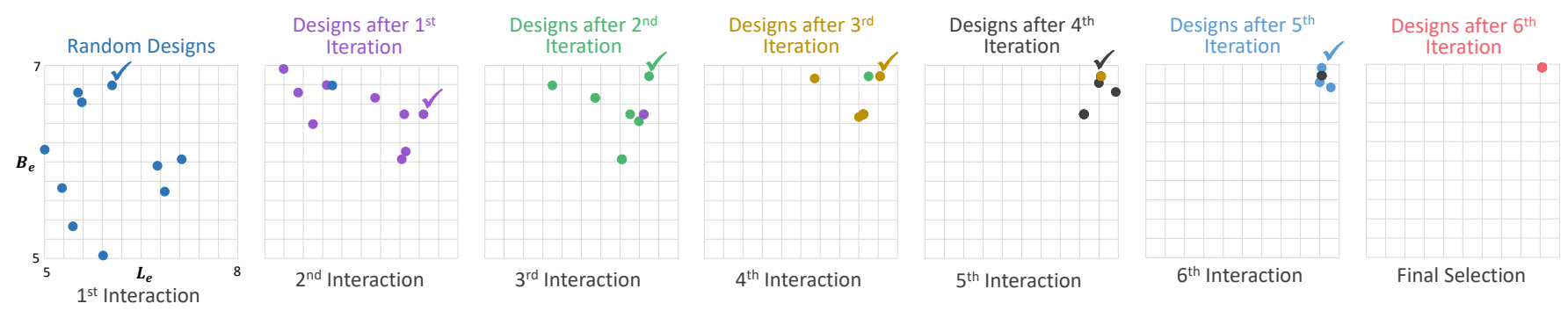

Figure 16: Interactive results of the interactive genetic algorithm (IGA). The design space was created using two geometric parameters $L_{e}$ and $B_{e}$. Designs were first created for the first interaction. Genetic algorithm (GA) then performed an iteration to generate a new population for the next interaction, while converging towards the selected design. The interactive process was repeated until all the designs were converged to the preferred one (see the last image).
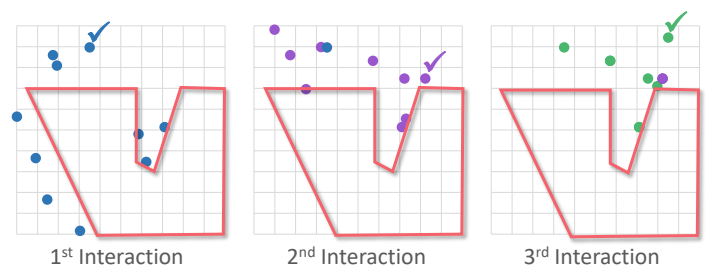

Figure 17: The area bounded in red (of the design space in Figure 16) was unexplored when IGA was used.

Marine Engineering, International Center for Numerical Methods in Engineering, 2017.

[9] A. Papanikolaou, Holistic ship design optimization, Computer-Aided Design 42 (11) (2010) 1028-1044.

[10] H. Cui, O. Turan, P. Sayer, Learning-based ship design optimization approach, Computer-Aided Design 44 (3) (2012) 186-195.

[11] S. Krish, A practical generative design method, Computer-Aided Design 43 (1) (2011) 88-100.

[12] S. Khan, M. J. Awan, A generative design technique for exploring shape variations, Advanced Engineering Informatics 38 (2018) 712-724.

[13] M. Bole, Interactive hull form transformations using curve network deformation, Ship Technology Research 58 (1) (2011) 46-64.

[14] A. Schulz, J. Xu, B. Zhu, C. Zheng, E. Grinspun, W. Matusik, Interactive design space exploration and optimization for cad models, ACM Transactions on Graphics (TOG) 36 (4) (2017) 157.

[15] A. Schulz, H. Wang, E. Crinspun, J. Solomon, W. Matusik, Interactive exploration of design trade-offs, ACM Transactions on Graphics (TOG) 37 (4) (2018) 131.

[16] N. Umetani, B. Bickel, Learning three-dimensional flow for interactive aerodynamic design, ACM Transactions on Graphics (TOG) 37 (4) (2018) 89.

[17] A. M. Brintrup, J. J. Ramsden, H. Takagi, Ergonomic chair design by fusing qualitative and quantitative criteria using interactive genetic algorithms.
[18] A. Mortazavi, V. Toğan, A. Nuhoğlu, Interactive search algorithm: A new hybrid metaheuristic optimization algorithm, Engineering Applications of Artificial Intelligence 71 (2018) 275-292.

[19] E. Poirson, J.-F. Petiot, L. Boivin, D. Blumenthal, Eliciting user perceptions using assessment tests based on an interactive genetic algorithm, Journal of Mechanical Design 135 (3) (2013) 031004.

[20] J. Felkner, E. Chatzi, T. Kotnik, Interactive truss design using particle swarm optimization and nurbs curves, Journal of Building Engineering 4 (2015) 60-74.

[21] R. Dou, C. Zong, G. Nan, Multi-stage interactive genetic algorithm for collaborative product customization, Knowledge-Based Systems 92 (2016) 43-54.

[22] S. Khan, E. Gunpinar, K. M. Dogan, A novel design framework for generation and parametric modification of yacht hull surfaces, Ocean Engineering 136 (2017) 243259.

[23] S. Chaudhuri, E. Kalogerakis, S. Giguere, T. Funkhouser, Attribit: content creation with semantic attributes, in: Proceedings of the 26th annual ACM symposium on User interface software and technology, ACM, 2013, pp. 193202.

[24] G. Nishida, I. Garcia-Dorado, D. G. Aliaga, B. Benes, A. Bousseau, Interactive sketching of urban procedural models, ACM Transactions on Graphics (TOG) 35 (4) (2016) 130.

[25] K. Liu, X. Zeng, P. Bruniaux, X. Tao, X. Yao, V. Li, J. Wang, 3d interactive garment pattern-making technology, Computer-Aided Design 104 (2018) 113-124.

[26] N. Umetani, Exploring generative 3d shapes using autoencoder networks, in: SIGGRAPH Asia 2017 Technical Briefs, ACM, 2017, p. 24.

[27] E. Gunpinar, S. E. Ovur, S. Gunpinar, A user-centered side silhouette generation system for sedan cars based on shape templates, Optimization and Engineering (2018) 141. 


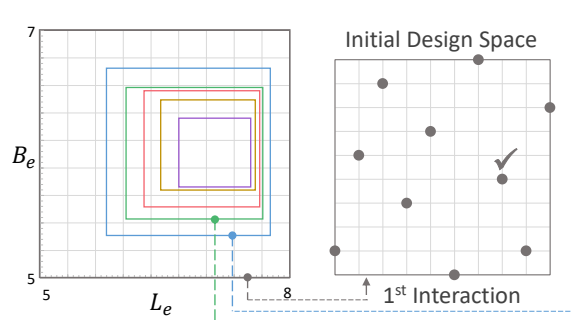

(a)

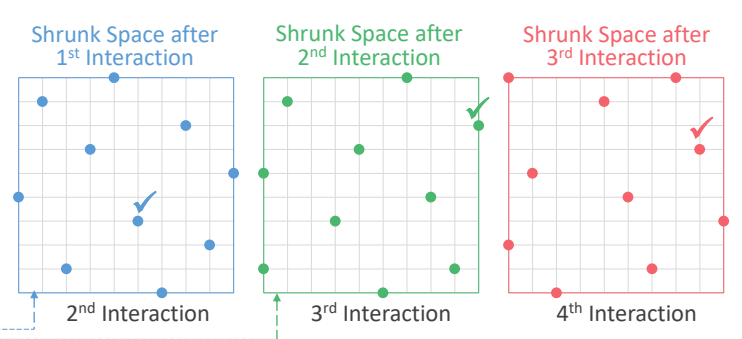

(b)

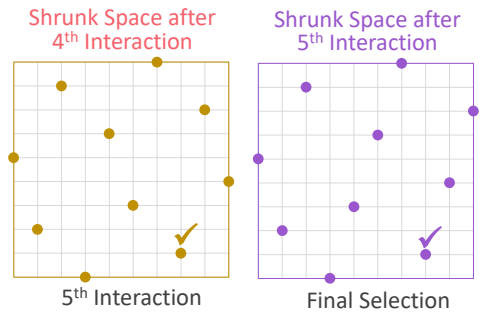

Figure 18: Interactive results when using GenYacht in the design space shown in Figure 16. Initial and shrunk design spaces in each interaction (a). Ten designs were generated using GDT in the interactions and the design space was shrunk using space shrinking technique (SST) based on the user selection at shrink rates of $\lambda_{-}=0.5$ for the first three and $\lambda_{-}=1.0$ for the last two interactions (b).

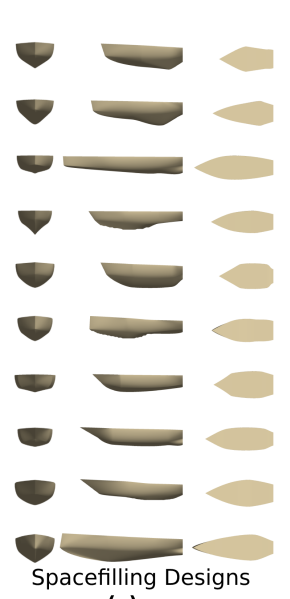

(a)

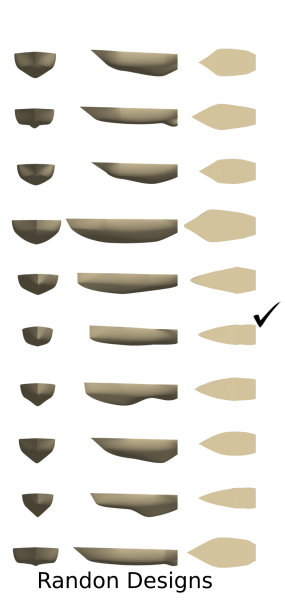

Randon Designs

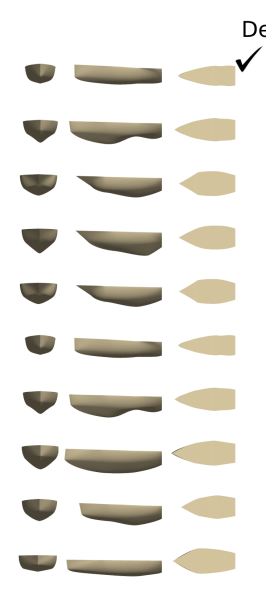

Designs Generated Using IGA

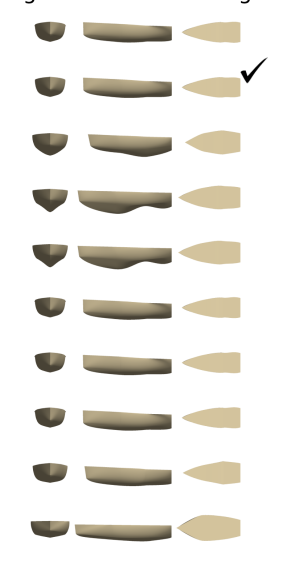

(b)

Figure 19: Hull forms generated using the GDT (a). Hull forms created during the interactive process performed using IGA (b). Designs converged (i.e., got similar) at the fourth interaction without significant diversifications in the hull models. Moreover, the left-most image of (b) shows the randomly generated designs, which are similar to each other compared to designs generated using GDT.

[28] S. Wang, H. Takagi, Improving the performance of predicting users' subjective evaluation characteristics to reduce their fatigue in iec, Journal of physiological anthropology and applied human science 24 (1) (2005) 81-85.

[29] R. Dou, C. Zong, M. Li, An interactive genetic algorithm with the interval arithmetic based on hesitation and its application to achieve customer collaborative product configuration design, Applied Soft Computing 38 (2016) 384-394.

[30] L. García-Hernández, H. Pierreval, L. Salas-Morera, A. Arauzo-Azofra, Handling qualitative aspects in unequal area facility layout problem: An interactive genetic algorithm, Applied Soft Computing 13 (4) (2013) 17181727.

[31] A. T. Machwe, I. C. Parmee, Reducing user fatigue within an interactive evolutionary design system using clustering and case-based reasoning, Engineering Optimization 41 (9) (2009) 871-887.

[32] Z. Gu, M. X. Tang, J. H. Frazer, Capturing aesthetic intention during interactive evolution, Computer-Aided Design 38 (3) (2006) 224-237.

[33] O. Bandte, A broad and narrow approach to interactive evolutionary design — an aircraft design example, Applied Soft Computing 9 (1) (2009) 448-455.

[34] A. Ramírez, J. R. Romero, S. Ventura, Interactive multiobjective evolutionary optimization of software architectures, Information Sciences 463 (2018) 92-109.

[35] R. A. Danhaive, C. T. Mueller, Combining parametric modeling and interactive optimization for highperformance and creative structural design, in: Proceedings of IASS Annual Symposia, Vol. 2015, International Association for Shell and Spatial Structures (IASS), 2015, pp. 1-11.

[36] J. Zheng, G. Yu, Q. Zhu, X. Li, J. Zou, On decomposition methods in interactive user-preference based optimization, Applied Soft Computing 52 (2017) 952-973.

[37] T. W. DeNucci, Capturing design: Improving conceptual ship design through the capture of design rationale, Ph.D. thesis, Delft University of Technology (2012).

[38] M. Diez, D. Peri, Robust optimization for ship conceptual design, Ocean Engineering 37 (2010) 966-977.

[39] Autodesk, Project fractal (2018). URL https://home.fractal.live/ 
[40] J. Matejka, M. Glueck, E. Bradner, A. Hashemi, T. Grossman, G. Fitzmaurice, Dream lens: Exploration and visualization of large-scale generative design datasets, in: Proceedings of the 2018 CHI Conference on Human Factors in Computing Systems, ACM, 2018, p. 369.

[41] S. Khan, E. Gunpinar, M. Moriguchi, H. Suzuki, Evolving a psycho-physical distance metric for generative design exploration of diverse shapes, Journal of Mechanical Design (2019) 1-16.

[42] R. H. Kazi, T. Grossman, H. Cheong, A. Hashemi, G. Fitzmaurice, Dreamsketch: Early stage 3d design explorations with sketching and generative design, in: Proceedings of the 30th Annual ACM Symposium on User Interface Software and Technology, ACM, 2017, pp. 401414.

[43] L. Zaman, W. Stuerzlinger, C. Neugebauer, R. Woodbury, M. Elkhaldi, N. Shireen, M. Terry, Gem-ni: A system for creating and managing alternatives in generative design, in: Proceedings of the 33rd Annual ACM Conference on Human Factors in Computing Systems, ACM, 2015, pp. 1201-1210.

[44] L. Zaman, W. Stuerzlinger, C. Neugebauer, Mace: A new interface for comparing and editing of multiple alternative documents for generative design, in: Proceedings of the 2017 ACM Symposium on Document Engineering, ACM, 2017, pp. 67-76.

[45] E. Gunpinar, S. Gunpinar, A shape sampling technique via particle tracing for cad models, Graphical Models 96 (2018) 11-29.

[46] M. Turrin, P. Von Buelow, R. Stouffs, Design explorations of performance driven geometry in architectural design using parametric modeling and genetic algorithms, Advanced Engineering Informatics 25 (4) (2011) 656-675.

[47] P. Janssen, Dexen: A scalable and extensible platform for experimenting with population-based design exploration algorithms, AI EDAM 29 (4) (2015) 443-455.

[48] L. Caldas, Generation of energy-efficient architecture solutions applying gene_arch: An evolution-based generative design system, Advanced Engineering Informatics 22 (1) (2008) 59-70.

[49] S. Khan, E. Gunpinar, Sampling cad models via an extended teaching-learning-based optimization technique, Computer-Aided Design 100 (2018) 52-67.

[50] P. Audze, New approach to planning out of experiments, Problems of dynamics and strengths 35 (1977) 104-107.

[51] R. L. Haupt, S. E. Haupt, S. E. Haupt, Practical genetic algorithms, Vol. 2, Wiley New York, 1998.

[52] J. Kennedy, Particle swarm optimization, in: Encyclopedia of machine learning, Springer, 2011, pp. 760-766.
[53] D. Karaboga, B. Basturk, On the performance of artificial bee colony (abc) algorithm, Applied soft computing 8 (1) (2008) 687-697.

[54] R. V. Rao, V. J. Savsani, D. Vakharia, Teaching-learningbased optimization: a novel method for constrained mechanical design optimization problems, Computer-Aided Design 43 (3) (2011) 303-315.

[55] R. V. Rao, G. Waghmare, A new optimization algorithm for solving complex constrained design optimization problems, Engineering Optimization 49 (1) (2017) 60-83.

[56] K. Deb, A. Pratap, S. Agarwal, T. Meyarivan, A fast and elitist multiobjective genetic algorithm: Nsga-ii, IEEE transactions on evolutionary computation 6 (2) (2002) 182-197.

[57] J. Keuning, M. Katgert, A bare hull resistance prediction method derived from the results of the delft systematic yacht hull series extended to higher speeds, in: International Conference on Innovation in High Performance Sailing Yachts, Lorient, France, 2008.

[58] E. V. Lewis, Principles of naval architecture second revision, Jersey: SNAME 2. 